Geometry \& $\mathcal{T}$ opology

Volume 7 (2003) 399-441

Published: 24 June 2003

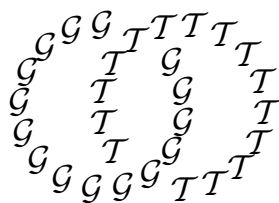

\title{
The virtual Haken conjecture: Experiments and examples
}

\author{
NATHAN M DUNFIELD \\ William P THURSTON \\ Department of Mathematics, Harvard University \\ Cambridge MA, 02138, USA \\ and \\ Department of Mathematics, University of California, Davis \\ Davis, CA 95616, USA \\ Email: nathand@math.harvard.edu and wpt@math.ucdavis.edu
}

\begin{abstract}
A 3-manifold is Haken if it contains a topologically essential surface. The Virtual Haken Conjecture says that every irreducible 3-manifold with infinite fundamental group has a finite cover which is Haken. Here, we discuss two interrelated topics concerning this conjecture.

First, we describe computer experiments which give strong evidence that the Virtual Haken Conjecture is true for hyperbolic 3-manifolds. We took the complete HodgsonWeeks census of 10,986 small-volume closed hyperbolic 3-manifolds, and for each of them found finite covers which are Haken. There are interesting and unexplained patterns in the data which may lead to a better understanding of this problem.

Second, we discuss a method for transferring the virtual Haken property under Dehn filling. In particular, we show that if a 3-manifold with torus boundary has a Seifert fibered Dehn filling with hyperbolic base orbifold, then most of the Dehn filled manifolds are virtually Haken. We use this to show that every non-trivial Dehn surgery on the figure- 8 knot is virtually Haken.
\end{abstract}

AMS Classification numbers Primary: 57M05, 57M10

Secondary: 57M27, 20E26, 20F05

Keywords: Virtual Haken Conjecture, experimental evidence, Dehn filling, onerelator quotients, figure- 8 knot

Proposed: Jean-Pierre Otal

Seconded: Walter Neumann, Martin Bridson

Received: 30 September 2002

Accepted: 13 April 2003

(C) Geometry $\& \mathcal{G}$ Topology $\mathcal{P}$ ublications 


\section{Introduction}

Let $M$ be an orientable 3-manifold. A properly embedded orientable surface $S \neq S^{2}$ in $M$ is incompressible if it is not boundary parallel, and the inclusion $\pi_{1}(S) \rightarrow \pi_{1}(M)$ is injective. A manifold is Haken if it is irreducible and contains an incompressible surface. Haken manifolds are by far the best understood class of 3-manifolds. This is because splitting a Haken manifold along an incompressible surface results in a simpler Haken manifold. This allows induction arguments for these manifolds.

However, many irreducible 3-manifolds with infinite fundamental group are not Haken, e.g. all but 4 Dehn surgeries on the figure- 8 knot. It has been very hard to prove anything about non-Haken manifolds, at least without assuming some sort of additional Haken-like structure, such as a foliation or lamination.

Sometimes, a non-Haken 3-manifold $M$ has a finite cover which is Haken. Most of the known properties for Haken manifolds can then be pushed down to $M$ (though showing this can be difficult). Thus, one of the most interesting conjectures about 3-manifolds is Waldhausen's conjecture [54]:

1.1 Virtual Haken Conjecture Suppose $M$ is an irreducible 3-manifold with infinite fundamental group. Then $M$ has a finite cover which is Haken.

A 3-manifold satisfying this conjecture is called virtually Haken. For more background and references on this conjecture see Kirby's problem list [38], problems $3.2,3.50$, and 3.51. See also $[12,13]$ and $[40,39]$ for some of the latest results toward this conjecture. The importance of this conjecture is enhanced because it's now known that 3-manifolds which are virtually Haken are geometrizable $[27,26,49,41,42,25,9]$.

There are several stronger forms of this conjecture, including asking that the finite cover be not just Haken but a surface bundle over the circle. We will be interested in the following version. Let $M$ be a closed irreducible 3-manifold. If $H_{2}(M, \mathbb{Z}) \neq 0$ then $M$ is Haken, as any non-zero class in $H_{2}(M, \mathbb{Z})$ can be represented by an incompressible surface. Now $H_{2}(M, \mathbb{Z})$ is isomorphic to $H^{1}(M, \mathbb{Z})$ by Poincaré duality, and $H^{1}(M, \mathbb{Z})$ is a free abelian group. So if the first betti-number of $M$ is $\beta_{1}(M)=\operatorname{dim} H_{1}(M, \mathbb{R})=\operatorname{dim} H^{1}(M, \mathbb{R})$, then $\beta_{1}(M)>0$ implies $M$ is Haken. As the cover of an irreducible 3-manifold is irreducible [41], a stronger form of the Virtual Haken Conjecture is:

1.2 Virtual Positive Betti Number Conjecture Suppose $M$ is an irreducible 3-manifold with infinite fundamental group. Then $M$ has a finite cover $N$ where $\beta_{1}(N)>0$. 
We will say that such an $M$ has virtual positive betti number. Note that $\beta_{1}(N)>0$ if and only if $H_{1}(N, \mathbb{Z})$, the abelianization of $\pi_{1}(N)$, is infinite. So an equivalent, more algebraic, formulation of Conjecture 1.2 is:

1.3 Conjecture Suppose $M$ is an irreducible 3-manifold. Assume that $\pi_{1}(M)$ is infinite. Then $\pi_{1}(M)$ has a finite index subgroup with infinite abelianization.

Here, we focus on this form of the Virtual Haken Conjecture because its algebraic nature makes it easier to examine both theoretically and computationally. While in theory one can to use normal surface algorithms to decide if a manifold is Haken [37], in practice these algorithms are prohibitively slow in all but the simplest examples. Computing homology is much easier as it boils down to computing the rank of a matrix. Also, it's probably true that having virtual positive betti number isn't much stronger than being virtually Haken (see the discussion of [40] in Section 11 below).

\subsection{Outline of the paper}

This paper examines the Virtual Haken Conjecture in two interrelated parts:

\section{Experiment: Sections 2-6}

Here, we describe experiments which strongly support the Virtual Positive Betti Number Conjecture. We looked at the 10,986 small-volume hyperbolic manifolds in the Hodgson-Weeks census, and tried to show that they had virtual positive betti number. In all cases, we succeeded. It was natural to restrict to hyperbolic 3-manifolds for our experiment since, in practice, all 3-manifolds are geometrizable and the Virtual Positive Betti Number Conjecture is known for all other kinds of geometrizable 3-manifolds.

Section 2 gives an overview of the experiment and discusses the results and limitations of the survey. Sections 3 and 4 describe the techniques used to compute the homology of the covers. Section 5 discusses some interesting patterns that we found among the covers where the covering group is a simple group. Some further questions are given in Section 6.

\section{Examples and Dehn filling: Sections 7 - 12}

Here we consider Dehn fillings of a fixed 3-manifold $M$ with torus boundary. Generalizing work of Boyer and Zhang [5], we give a method for transferring 
virtual positive betti number from one filling of $M$ to another. Roughly, Theorem 7.3 says that if $M$ has a filling which is Seifert fibered with hyperbolic base orbifold, then most Dehn fillings have virtual positive betti number. We use this to give new examples of manifolds $M$ where all but finitely many Dehn fillings have virtual positive betti number. In Section 9, we show this holds for most surgeries on one component of the Whitehead link.

In the case of figure- 8 knot, we use work of Holt and Plesken [35] to amplify our results, and prove that every non-trivial Dehn surgery on the figure- 8 knot has virtual positive betti number (Theorem 10.1).

In Section 11, we discuss possible avenues to other results using fillings which are Haken rather than Seifert fibered. This approach is easiest in the case of toroidal Dehn fillings, and using these techniques we prove (Theorem 12.1) that all Dehn fillings on the sister of the figure- 8 complement satisfy the Virtual Positive Betti Number Conjecture.

\section{Acknowledgments}

The first author was partially supported by an NSF Postdoctoral Fellowship. The second author was partially supported by NSF grants DMS-9704135 and DMS-0072540. We would like to thank Ian Agol, Daniel Allcock, Matt Baker, Danny Calegari, Greg Kuperberg, Darren Long, Alex Lubotzky, Alan Reid, William Stein, and Dylan Thurston for useful conversations. We also thank of the authors of the computer programs SnapPea [56] and GAP [28] which were critical for our computations.

\section{The experiment}

\subsection{The manifolds}

We looked at the 10,986 hyperbolic 3-manifolds in the Hodgson-Weeks census of small-volume closed hyperbolic 3-manifolds [56]. The volumes of these manifolds range from that of the smallest known manifold $(0.942707 \ldots)$ to 6.5 . While there are infinitely many closed hyperbolic 3-manifolds with volume less than 6.5 , there are only finitely many if we also bound the injectivity radius from below. The census manifolds are an approximation to all closed hyperbolic 3 -manifolds with volume $<6.5$ and injectivity radius $>0.3$.

A more precise description of these manifolds is this. Start with the CallahanHildebrand-Weeks census of cusped finite-volume hyperbolic 3-manifolds, which 
is a complete list of the those having ideal triangulations with 7 or fewer tetrahedra $[34,8]$. The closed census consists of all the Dehn fillings on the 1-cusped manifolds in the cusped census, where the closed manifold has shortest geodesic of length $>0.3$.

Only 132 of the 10,986 manifolds have positive betti number. It is also worth mentioning that many (probably the vast majority) of these manifolds are nonHaken. For the 246 manifolds with volume less than 3, exactly 15 are Haken [19].

\subsection{Computational framework}

For each 3-manifold, we started with a finite presentation of its fundamental group $G$, and then looked for a finite index subgroup $H$ of $G$ which has infinite abelianization. There is a fair amount of literature on how find such an $H$, because finding a finite index subgroup with infinite abelianization is one of the main computational techniques for proving that a given finitely presented group is infinite. See [43] for a survey. The key idea which simplifies the computations is contained in [35], which we used in the form described in Section 3.

We used SnapPea [56] to give presentations for the fundamental groups of each of the manifolds in the closed census. We then used GAP [28] to find various finite index subgroups and compute the homology of the subgroups (see Sections 3-4).

\subsection{Types of covers}

When looking for a subgroup with positive betti number, we tried a number of different types of subgroups. Some types were much better at producing homology than others. Those that worked well were:

- Abelian/p-group covers with exponent 2 or 3 .

- Low $(<20)$ index subgroups. Coset enumeration techniques allow one to enumerate low-index subgroups [52]. Given such a subgroup $H<G$, we looked at the largest normal subgroup contained in $H$, to maximize the chance of finding homology.

- Normal subgroups where the quotient is a finite simple group. These were found by choosing the simple group in advance and then finding all epimorphisms of $G$ onto that group.

The following types were inefficient in producing homology:

- Abelian/nilpotent covers with exponents $>3$.

- Dihedral covers. 
- Intersections of subgroups of the types listed in the first list (the useful types).

It would be nice to have heuristics which explain why some things worked and others didn't (we plan to explore this further in [21]). Also, while intersecting subgroups was not efficient in general, there were certain manifolds where the only positive betti number cover we could find were of this type.

\subsection{Results}

We were able to find positive betti number covers for all of the Hodgson-Weeks census manifolds. For most of the manifolds, it was easy to find such a cover. For instance, just looking at abelian covers and subgroups of index $\leq 6$ works for $42 \%$ of the manifolds. See Table 1 for more about the degrees of the covers we used.

\begin{tabular}{rr}
$d$ & $\%$ \\
\hline 1 & 1.2 \\
2 & 3.8 \\
5 & 21.2 \\
6 & 39.3 \\
10 & 57.9 \\
20 & 68.3 \\
50 & 88.8 \\
100 & 95.6 \\
200 & 98.1
\end{tabular}

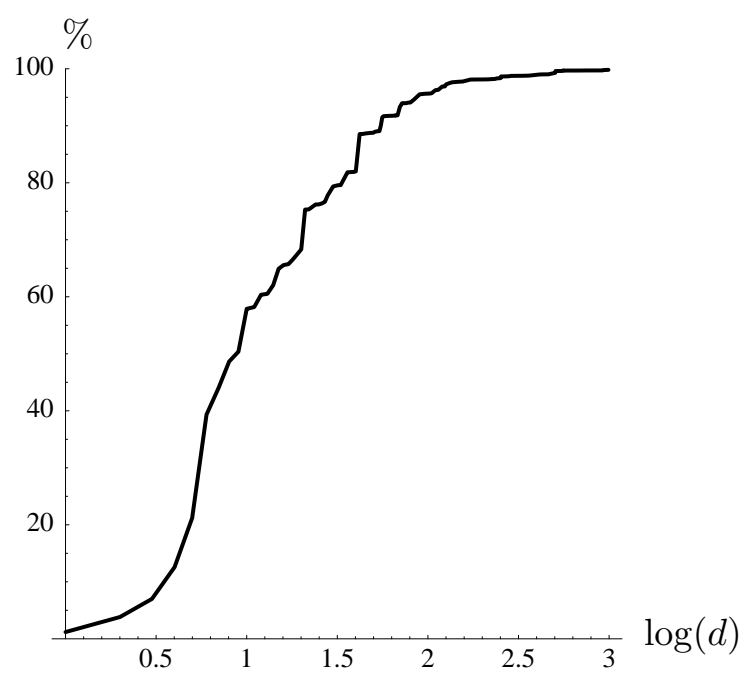

Table 1: The table at left shows the proportion of manifolds for which we found a cover with positive betti number of degree $\leq d$. Note this is just for the covers that we found, which are not always the positive betti number covers of smallest degree. The plot at right presents all of the data, where $\log (d)$ is base 10 .

For each of the manifolds, we stored a presentation of the fundamental group and a homomorphism from that finitely presented group to $S_{n}$ whose kernel has positive betti number. This information is available on the web at [20] together with the GAP code we used for the computations, and will hopefully be useful as a source of examples. The amount of computer time used to find all the covers 
was in excess of one CPU-year, but the amount of time needed to check all the covers for homology given the data available at [20] is only a few of hours.

There was one manifold in particular where it was very difficult to find a cover with positive betti number. This manifold is $N=s 633(2,3)$. Its volume is $4.49769817315 \ldots$ and $H_{1}(N)=\mathbb{Z} / 79 \mathbb{Z}$. The manifold $N$ has a genus-2 Heegaard splitting, and is the 2-fold branched cover of the 3-bridge knot in Figure 1 . One of the reasons that $N$ was so difficult is that $\pi_{1}(N)$ has very

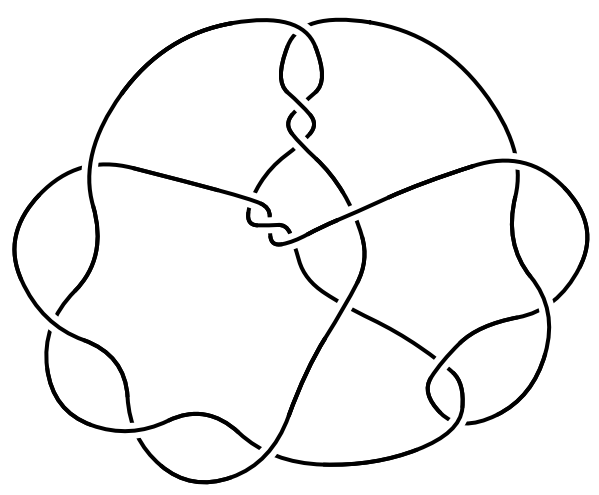

Figure 1: The 2-fold cover branched over this knot is the manifold $N$. Figure created with [36].

few low-index subgroups (the smallest index is 13). In the end, a search using Magma [4], turned up a subgroup of index 14 which has positive betti number. It is very hard to enumerate all finite-index subgroups for an index as large as 14 , roughly because the size of $S_{n}$ is $n$ !; finding this index 14 subgroup took 2 days of computer time.

While $\pi_{1}(N)$ has few subgroups of low index, it does have a reasonable number of simple quotients, and might be a good place to look for a co-final sequence of covers which fail to have positive betti number. The manifold $N$ is nonHaken, but it contains a essential lamination (and thus a genuine lamination [7]). Arithmetically, it is quite a complicated manifold - Snap [29] computes that the trace field has a minimal polynomial $p(x)$ whose degree is 51 and largest coefficient is about $4 \times 10^{7}$. The coefficients of $p$ are, starting with the constant term:

1, 24, 223, 929, 909, -6163, -20232, -2935, 79745, 121259, -57077, -428280,

$-507427,689749,2245466,-519994,-5455251,355551,9513149,-1958013$, 
$-12213255,7478063,10535124,-17696676,-4109720,30159462,-2803266$, $-39076707,5291640,39199917,-3032906,-30650313,-365203,18711624$, $1997701,-8892931,-1776338,3259601,951237,-903591,-352258,182336$, $93101,-24677,-17396,1748,2197,33,-169,-17,6,1$.

\subsection{Overlap with known results}

The manifolds we examined have little overlap with those covered by the known results about the Virtual Haken Conjecture. The only general results are those of Cooper and Long [12, 13] building on work of Freedman and Freedman [24]. These are Dehn surgery results - they say that many "large" Dehn fillings on a 1-cusped hyperbolic 3-manifold are virtually Haken. Because "large" Dehn fillings usually have short geodesics, the Cooper-Long results probably apply to very few, if any, of the census manifolds.

\subsection{Limitations}

It's possible the behavior we found might not be true in general because the census manifolds are non-generic in a couple ways. First, they all have fundamental groups with presentations with at most 3 generators. About $75 \%$ have 2-generator presentations. For these manifolds, it seems that (at least most of the time) the number of generators and the Heegaard genus coincide. So most of these manifolds have Heegaard genus 2 or 3.

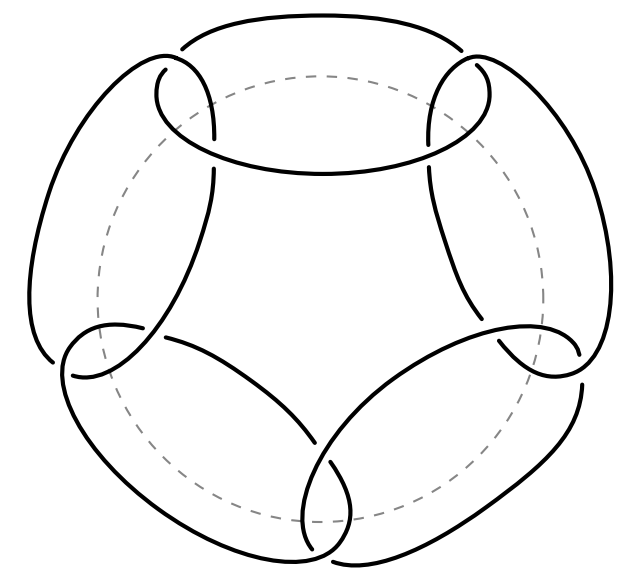

Figure 2: The minimally-twisted 5-chain link.

Moreover Callahan, Hodgson, and Weeks (unpublished) showed that almost all of the census manifolds are Dehn surgeries on a single 5-component link, the 
minimally twisted 5-chain shown in Figure 2. Let $L$ be this link and $M=$ $S^{3} \backslash N(L)$ be its exterior. The link $L$ is invariant under rotation of $\pi$ about the dotted grey axis. The induced involution of $M$ acts on each torus in $\partial M$ by the elliptic involution. Thus the involution of $M$ extends to an orientation preserving involution of every Dehn filling of $M$. So almost all of the census manifolds have an orientation preserving involution where the fixed point set is a link and underlying space of the quotient is $S^{3}$. While any manifold which has a genus-2 Heegaard splitting has such an involution [3], this says that the other $25 \%$ of the census manifolds are also special. The presence of such an involution has proven useful in the past. For instance, it implies that the manifold is geometrizable. So it's possible that our computations only reflect the situation for manifolds of this type.

The 5-chain $L$ is a truly beautiful link, and it's worth describing some of its properties here. The orbifold $N$ which is $M$ modulo this involution is easy to describe. Take the triangulation $\mathcal{T}$ of $S^{3}$ gotten by thinking of $S^{3}$ as the boundary of the 4-simplex. The 1-skeleton of $\mathcal{T}$ is called the pentacle, see Figure 3. If we take $S^{3}$ minus an open ball about each vertex in $\mathcal{T}$, and label

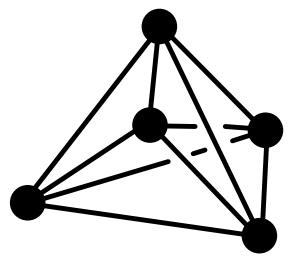

Figure 3: The pentacle.

what's left of each edge of the pentacle by $\mathbb{Z} / 2 \mathbb{Z}$, we get exactly the orbifold $N$ !

We can put a hyperbolic structure on $N$ and thus $M$ by making each tetrahedron in $\mathcal{T}$ a regular ideal tetrahedron. Thus the volume of $M$ is $10 v_{3}=$ $10.149416064 \ldots$, and further $M$ is arithmetic and commensurable with the Bianchi group $\mathrm{PSL}_{2} \mathrm{O}(\sqrt{-3})$. The symmetric group $S_{5}$ acts on the 4 -simplex by permuting the vertices, inducing an action of $S_{5}$ on $N$. This action is exactly the group of isometries of $N$. The isometry group of $M$ is $S_{5} \times \mathbb{Z} / 2 \mathbb{Z}$, where the $\mathbb{Z} / 2 \mathbb{Z}$ is the rotation about the axis.

The manifold $M$ fibers over the circle, and in fact every face of the Thurston norm ball is fibered. Here's an explicit way to see that $N$ fibers over the interval $I$ with mirrored endpoints (this fibration lifts to a fibration of $M$ over $S^{1}$ ). Take 
any Hamiltonian cycle in the 1 -skeleton of $\mathcal{T}$. The complementary edges also form a Hamiltonian cycle. Split the fat vertices of $\mathcal{T}$ (the cusps of $N$ ) in the obvious way in space so that these two cycles become the unlink, with cusps stretched between them. Then the special fibers over the $\mathbb{Z} / 2 \mathbb{Z}$ endpoints of $I$ are two pentagons, spanning the two Hamiltonian cycles. The other fibers are 5 -punctured spheres.

\section{Techniques for computing homology}

Given a finite index subgroup $H$ of a finitely presented group $G$, a simplified version of the Reidemeister-Schreier method produces a matrix $A$ with integers entries whose cokernel is the abelianization of $H$. Computing this matrix is not very time-consuming. The hard part of computing the rank of the abelianization of $H$ is finding the rank of $A$. Computing the rank of a matrix is $O\left(n^{3}\right)$ if field operations are constant time. We need to compute the rank over $\mathbb{Q}$ so the time needed is somewhat more than that (see Section 4). The side lengths of $A$ are usually about $n=[G: H]$, which at $O\left(n^{3}\right)$ is prohibitive for many of the covers that we looked at (the largest covering group we needed was $\mathrm{PSL}_{2} \mathbb{F}_{101}$, whose order is 515,100$)$.

So one wants to keep the degree of the cover, or really the size of the matrix involved, as small as possible. One way to do this, first used in this context by Holt and Plesken [35], is the following application of the representation theory of finite groups. Suppose $H$ is a finite index subgroup of $G$. Assume that $H$ is normal, so the corresponding cover is regular. Set $Q=G / H$ and let $f: G \rightarrow Q$ be the quotient map. The group $Q$ acts on the homology of the cover $H_{1}(H, \mathbb{C})$, giving a representation of $Q$ on the vector space $H_{1}(H, \mathbb{C})$. Another description of $H_{1}(H, \mathbb{C})$ is that it is the homology with twisted coefficients $H_{1}(G, \mathbb{C} Q)$. As a $Q$-module, $\mathbb{C} Q$ decomposes as $\mathbb{C} Q=V_{1}^{n_{1}} \oplus V_{2}^{n_{2}} \oplus \cdots \oplus V_{k}^{n_{k}}$ where the $V_{i}$ are simple $Q$-modules and $\operatorname{dim} V_{i}=n_{i}$. So

$$
H_{1}(H)=H_{1}(G, \mathbb{C} Q)=H_{1}\left(G, V_{1}\right)^{n_{1}} \oplus H_{1}\left(G, V_{2}\right)^{n_{2}} \oplus \cdots \oplus H_{1}\left(G, V_{k}\right)^{n_{k}} .
$$

Since the dimensions of the $V_{i}$ are usually much less than the order of $Q$, the matrices involved in computing $H_{1}\left(G, V_{i}\right)$ are much smaller than the one you would get by applying Reidemeister-Schreier to the subgroup $H$. For instance, $\mathrm{PSL}_{2} \mathbb{F}_{p}$ has order about $(1 / 2) p^{3}$, but every $V_{i}$ has dimension about $p$. If we want to show that $H_{1}(H, \mathbb{C})$ is non-zero, we just have to compute that a single $H_{1}\left(G, V_{i}\right)$ is non-zero.

There are a couple of difficulties in computing $H_{1}\left(G, V_{i}\right)$. First, to do the computation rigorously, we need to compute not over $\mathbb{C}$ but over a finite extension 
of $\mathbb{Q}$. Now there is a field $k$ so that $k Q$ splits over $k$ the same way as $\mathbb{C} Q$ splits over $\mathbb{C}$. However, the matrices we need to compute $H_{1}\left(G, V_{i}\right)$ will have entries in $k$, whereas the matrix given to us by Reidemeister-Schreier has integer entries. If $A$ is a matrix with entries in $k$, to compute its rank over $\mathbb{Q}$ one can form an associated $\mathbb{Q}$-matrix $B$ by embedding $k$ as a subalgebra of $\mathrm{GL}_{n} \mathbb{Q}$ where $n$ is $[k: \mathbb{Q}]$ (see e.g. [45]). The rank of $B$ can then be computed using one the techniques for integer matrices. However, the size of $B$ is the size of $A$ times $[k: \mathbb{Q}]$, so this eats up part of the apparent advantage to computing just the $H_{1}\left(G, V_{i}\right)$.

The other problem is that we may not know what the irreducible representations of $Q$ are, especially if we don't know much about $Q$. While computing the character table of a finite group is a well-studied problem, the problem of finding the actual representations is harder and not one of the things that GAP or other standard programs can do. Even when the representations of $Q$ are explicitly known (e.g. $Q=\mathrm{PSL}_{2} \mathbb{F}_{p}$ ), it can be time-consuming to tell the computer how to construct the representations. For more on computing the actual representations see $[16,44]$.

We used the following modified approach which avoids the two difficulties just mentioned, while still reducing the size of the matrices considerably. Suppose we are given normal subgroup $H$ and we want to determine if $H_{1}(H, \mathbb{C})$ is non-zero. Suppose $U$ is a subgroup of $Q$. Note $U$ is not assumed to be normal. The permutation representation of $Q$ on $\mathbb{C}[Q / U]$ desums into irreducible representations, say $\mathbb{C}[Q / U]=V_{1}^{e_{1}} \oplus V_{2}^{e_{2}} \oplus \cdots \oplus V_{k}^{e_{k}}$. Let $K=f^{-1}(U)$, a finite index subgroup of $G$ containing $H$. Then

$$
H_{1}(K)=H_{1}(G, \mathbb{C}[Q / U])=H_{1}\left(G, V_{1}\right)^{e_{1}} \oplus H_{1}\left(G, V_{2}\right)^{e_{2}} \oplus \cdots \oplus H_{1}\left(G, V_{k}\right)^{e_{k}} .
$$

Suppose that $U$ is chosen so that every irreducible representation appears in $\mathbb{C}[Q / U]$, that is, every $e_{i}>0$. Then we see that $H_{1}(H)$ is non-zero if and only if $H_{1}(K)$ is. As long as $U$ is non-trivial, the index $[G: K]=[Q: U]$ is smaller than $[G: H]=\# Q$, so computing $H_{1}(K)$ is easier that computing $H_{1}(H)$. Returning to the example of $\mathrm{PSL}_{2} \mathbb{F}_{p}$, there is such a $U$ of index about $p^{2}$, whereas the order of $\mathrm{PSL}_{2} \mathbb{F}_{p}$ is about $p^{3} / 2$. Looking at a matrix with side $O\left(p^{2}\right)$ is a big savings over one of side $O\left(p^{3}\right)$.

Moreover, finding such a $U$ given $Q$ is easy. First compute the character table of $Q$ and the conjugacy classes of subgroups of $Q$ (these are both well-studied problems). For each subgroup $U$ of $Q$ compute the character $\chi_{U}$ of the permutation representation of $Q$ on $\mathbb{C}[Q / U]$. Expressing $\chi_{U}$ as a linear combination of the irreducible characters tells us exactly what the $e_{i}$ are. Running through the $U$, we can find the subgroup of lowest index where all of the $e_{i}>0$. 
When we were searching for positive betti number covers, we used this method of replacing $H$ with $K=f^{-1}(U)$ and computed the ranks of the resulting matrices over a finite field $\mathbb{F}_{p}$. Once we had found an $H$ with positive $\mathbb{F}_{p}$-betti number, we did the following to check rigorously that $H$ has infinite abelianization. First, we went through all the subgroups $U$ of $Q$, till we found the $U$ of smallest index such that $f^{-1}(U)$ has positive $\mathbb{F}_{p}$-betti number. For this $U$, we computed the $\mathbb{Q}$-betti number of $f^{-1}(U)$ using one of the methods described in Section 4. Doing this kept the matrices that we needed to compute the $\mathbb{Q}$-rank of small, and was the key to checking that the covers really had positive $\mathbb{Q}$-betti number. For instance, for the $\mathrm{PSL}_{2} \mathbb{F}_{101}$-cover of degree 515,100 there was a $U$ so that the intermediate cover $f^{-1}(U)$ with positive betti number had degree "only" 5,050.

It's worth mentioning that the rank over $\mathbb{Q}$ was very rarely different than that over a small finite field. Initially, for each manifold we found a cover where the

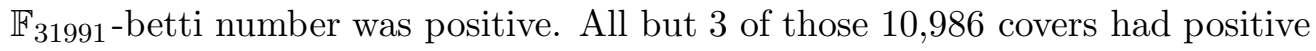
$\mathbb{Q}$-betti number.

\section{Computing the rank over $\mathbb{Q}$}

Here, we describe how we computed the $\mathbb{Q}$-rank of the matrices produced in the last section. Normally, one thinks of linear algebra as "easy", but standard row-reduction is polynomial time only if field operations are constant time. To compute the rank of an integer matrix $A$ rigorously one has to work over $\mathbb{Q}$. Here, doing row reduction causes the size of the fractions involved to explode. There are a number of ways to try to avoid this.

The first is to use a clever pivoting strategy to minimize the size of the fractions involved [33, 32, 31]. This is the method built into GAP, and was what we used for the covers of degree less than 500 , which sufficed for $99.2 \%$ of the manifolds.

For all but about 7 of the remaining 94 manifolds, we used a simplified version of the $p$-adic algorithm of Dixon given in [17]. Over a large finite field $\mathbb{F}_{p}$, we computed a basis of the kernel of the matrix. Then we used "rational reconstruction", a partial inverse to the map $\mathbb{Q} \rightarrow \mathbb{F}_{p}$ to try to lift each of the $\mathbb{F}_{p}$-vectors to $\mathbb{Q}$-vectors (see $[17$, pg. 139$]$ ). If we succeeded, we then checked that the lifted vectors were actually in the kernel over $\mathbb{Q}$.

For 7 of the largest covers (degree 1,000-5,000), this simplification of Dixon's algorithm fails, and we used the program MAGMA [4], which has a very sophisticated $p$-adic algorithm, to check the ranks of the matrices involved. 


\section{$5 \quad$ Simple covers}

To gain more insight into this problem, we looked at a range of simple covers for a randomly selected 1,000 of the census manifolds which have 2-generator fundamental groups. For these 1,000 manifolds we found all the covers where the covering group was a non-abelian finite simple group of order less than 33,000. For each cover we computed the homology. We will describe some interesting patterns we found.

First, look at Table 2. There, the simple groups are listed by their ATLAS [11] name (so, for instance, $L_{n}(q)=\mathrm{PSL}_{n} \mathbb{F}_{q}$ ), together with basic information about how many covers there are, and how many have positive betti number. There is quite a bit of variation among the different groups. For instance, only $11.3 \%$ of the manifold groups have $L_{2}(16)$ quotients but $42.8 \%$ have $L_{3}(4)$ quotients. Moreover, there are big differences in how successful the different kinds of covers are at producing homology. Only half of the $L_{2}(37)$ covers have positive betti number, but almost all $(97.5 \%)$ of the $U_{4}(2)$ covers do. There are no obvious reasons for these patterns (for instance, the success rates don't correlate strongly with the order of the group). It would be very interesting to have heuristics which explain them, and we will explore these issues in [21].

In terms of showing manifolds are virtually Haken, even the least useful group has a Hit rate greater than $10 \%$. That is, for any given group at least $10 \%$ of the manifolds have a positive betti number cover with that group. So unless things are strongly correlated between different groups, one would expect that every manifold would have a positive betti number simple cover, and that one would generally find such a cover quickly. Let $Q(n)$ denote the $n^{\text {th }}$ simple group as listed in Table 2. Set $V(n)$ to be the proportion of the manifolds which have a positive betti number $Q(k)$-cover where $k \leq n$. We expect that the increasing function $V(n)$ should rapidly approach 1 as $n$ increases. This is born out in Figure 4.

Figure 4 shows that the groups behave pretty independently of each other, although not completely as we will see. Let $H(n)$ denote the hit rate for $Q(n)$, that is the proportion of the manifolds with a $Q(n)$ cover with positive betti number. If everything were independent, then one would expect

$$
V(n) \approx V(n-1)+(1-V(n-1)) H(n) .
$$

If we let $E(n)$ be the right-hand side above, and compare $E(n)$ with $V(n)$ we find that $E(n)-V(n)$ is almost always positive. To judge the size of this 


$\begin{array}{rrrccc}\text { Quotient } & \text { Order } & \text { Hit } & \text { HavCov } & \text { SucRat1 } & \text { SucRat2 } \\ A_{5} & 60 & 14.0 & 26.9 & 52.0 & 52.9 \\ L_{2}(7) & 168 & 17.8 & 28.2 & 63.1 & 66.3 \\ A_{6} & 360 & 21.6 & 31.4 & 68.8 & 68.7 \\ L_{2}(8) & 504 & 15.4 & 21.7 & 71.0 & 72.6 \\ L_{2}(11) & 660 & 24.1 & 32.8 & 73.5 & 71.8 \\ L_{2}(13) & 1092 & 29.4 & 41.1 & 71.5 & 77.8 \\ L_{2}(17) & 2448 & 29.4 & 43.1 & 68.2 & 69.6 \\ A_{7} & 2520 & 41.1 & 45.8 & 89.7 & 90.9 \\ L_{2}(19) & 3420 & 28.2 & 44.4 & 63.5 & 65.7 \\ L_{2}(16) & 4080 & 11.3 & 18.3 & 61.7 & 65.3 \\ L_{3}(3) & 5616 & 19.2 & 28.0 & 68.6 & 76.5 \\ U_{3}(3) & 6048 & 16.4 & 18.0 & 91.1 & 92.8 \\ L_{2}(23) & 6072 & 32.7 & 47.6 & 68.7 & 70.1 \\ L_{2}(25) & 7800 & 24.7 & 33.0 & 74.8 & 75.5 \\ M_{11} & 7920 & 14.6 & 17.1 & 85.4 & 88.8 \\ L_{2}(27) & 9828 & 14.2 & 26.6 & 53.4 & 57.1 \\ L_{2}(29) & 12180 & 42.0 & 57.1 & 73.6 & 74.1 \\ L_{2}(31) & 14880 & 38.1 & 56.5 & 67.4 & 70.9 \\ A_{8} & 20160 & 18.7 & 20.7 & 90.3 & 92.3 \\ L_{3}(4) & 20160 & 42.8 & 50.2 & 85.3 & 89.1 \\ L_{2}(37) & 25308 & 24.9 & 54.2 & 45.9 & 50.5 \\ U_{4}(2) & 25920 & 26.6 & 27.8 & 95.7 & 97.5 \\ S z(8) & 29120 & 26.9 & 43.9 & 61.3 & 73.1 \\ L_{2}(32) & 32736 & 12.4 & 17.9 & 69.3 & 72.1\end{array}$

Table 2: Hit is the percentage of manifolds having a cover with this group which has positive betti number. HavCov is the percentage of manifolds having a cover with this group. SucRate1 is the percentage of manifolds having a cover with this group which have such a cover with positive betti number. SucRate2 is the percentage of covers with this group having positive betti number.

deviation, we look at

$$
\frac{E(n)-V(n)}{1-V(n-1)} \quad \text { which lies in }[-0.007,0.13]
$$

and which averages 0.022 . In other words, $V(n)-V(n-1)$ is usually about $2 \%$ smaller as a proportion of the possible increase than $E(n)-V(n-1)$. 


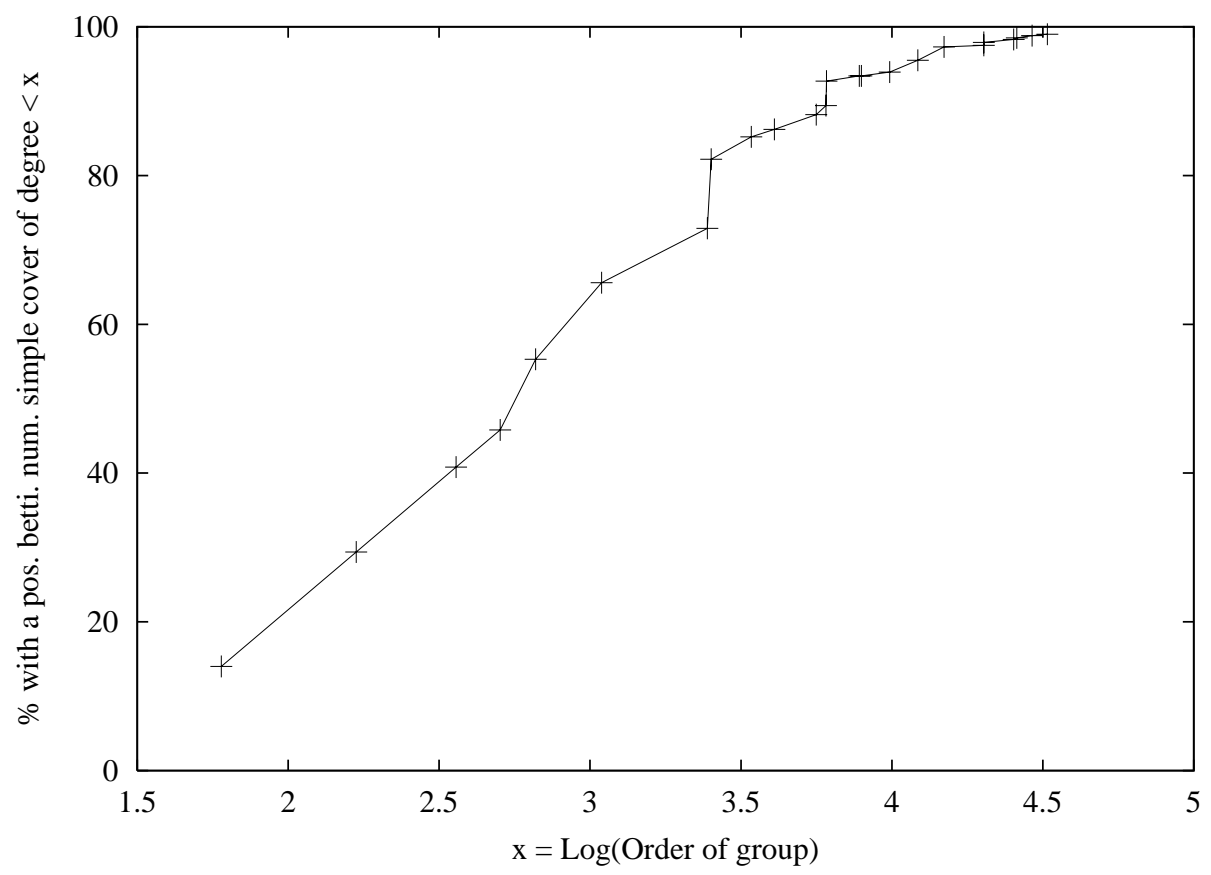

Figure 4: This graph shows how quickly simple group covers generate homology. Each + plotted is the pair $(\log (\# Q(n)), V(n))$, where the $\log$ is base 10 . Thus the leftmost + corresponds to the fact that $14 \%$ of the manifolds have an $A_{5}$ cover with positive betti number. The second leftmost + corresponds to the fact that $29 \%$ of the manifolds have either an $A_{5}$ or an $L_{2}(7)$ cover with positive betti number, and so on.

For a graphical comparison, define $V^{\prime}(n)$ by the recursion

$$
V^{\prime}(n)=V^{\prime}(n-1)+\left(1-V^{\prime}(n-1)\right) H(n),
$$

and compare with $V(n)$ in Figure 5.

Asymptotically, every non-abelian finite simple group is of the form $L_{2}(q)$, and so it's interesting to look at a modified $V(n)$ where we look only at the $Q(n)$ of this form. This is also shown in Figure 5.

\subsection{Amount of homology}

Suppose we look at a simple cover of degree $d$, what is the expected rank of the homology of the cover? The data suggests that the expected rank is linearly proportional to $d$. For the simple group $Q(n)$, set $R(n)$ to be the mean of $\beta_{1}(N)$, where $N$ runs over all the $Q(n)$ covers of our manifolds (including 


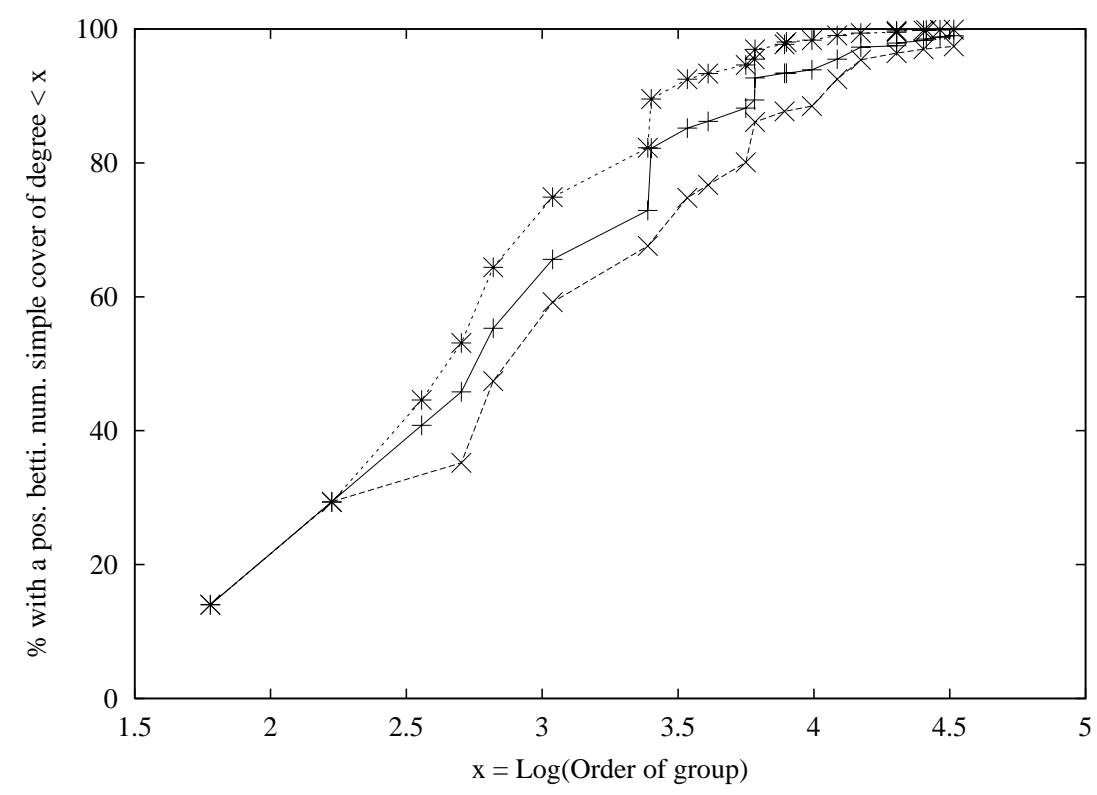

Figure 5: The top line plots $\left(\log (\# Q(n)), V^{\prime}(n)\right)$, the middle line $(\log (\# Q(n)), V(n))$ (as in Figure 4), and the lowest line plots only the groups of the form $L_{2}(q)$.

those where $\left.\beta_{1}(N)=0\right)$. Figure 6 gives a plot of $\log R(n)$ versus $\log (\# Q(n))$. Also shown is the line $y=x-1.3$ (which is almost the least squares fit line $y=1.018 x-1.303)$. The data points follow that line, suggesting that:

$$
\log R(n) \approx \log (\# Q(n))-1.3 \text { and hence } R(n) \approx \frac{\# Q(n)}{20} .
$$

Now each of the 3-manifold groups we are looking at here are quotients of the free group on two generators $F_{2}$. Let $G$ be fundamental group of one of our 3manifolds, say $G=F_{2} / N$. Given a homomorphism $G \rightarrow Q(n)$, we can look at the composite homomorphism $F_{2} \rightarrow Q(n)$. Let $H$ be the kernel of $G \rightarrow Q(n)$ and $K$ the kernel of $F_{2} \rightarrow Q(n)$. Then the rank of $H_{1}(K)$ is $\# Q(n)+1$. As $H_{1}(H)$ is a quotient of $H_{1}(K)$, Equation 1 is says that on average, $5 \%$ of $H_{1}(K)$ survives to $H_{1}(H)$.

This amount of homology is not a priori forced by the high hit rate for the $Q(n)$. For instance, $L_{2}(p)$ has order $\left(p^{3}-p\right) / 2$ but has a rational representation of dimension $p$. Thus it would be possible for $L_{2}(p)$ covers to have

$$
\log (R(n)) \approx(1 / 3) \log (\# G(n))+C,
$$

even if a large percentage of these covers had positive betti number. This data suggests that on a statistical level these 3 -manifold groups are trying to behave 


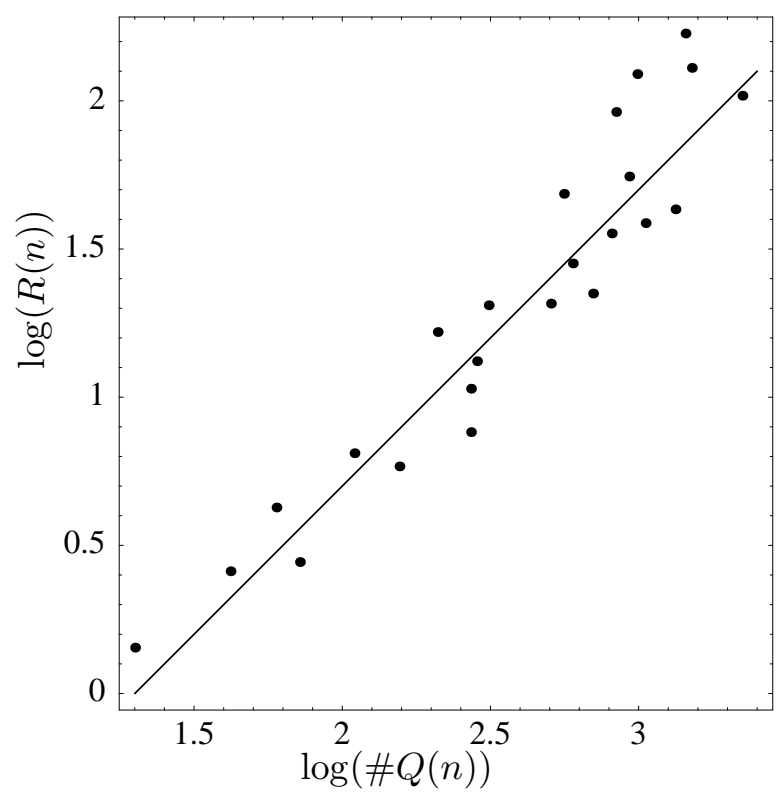

Figure 6: This plot shows the relationship between the expected rank and the degree of the cover. The line shown is $y=x-1.3$.

like the fundamental group of a 2-dimensional orbifold of Euler characteristic $-1 / 20$.

\section{Caveats}

The data in Figure 6 is not based on the full $Q(n)$ covers but on subcovers coming from a fixed subgroup $U(n)<Q(n)$, chosen as described in Section 3 . The degree plotted is the degree of the cover that was used, that is $[Q(n)$ : $U(n)$ ] not the order of $Q(n)$ itself, so the above analysis is still valid. Also, throughout Section 5 having positive betti number really means having positive betti number over $\mathbb{F}_{31991}$. Also, we originally used a list of the Hodgson-Weeks census which had a few duplicates and so there are actually 12 manifold which appear twice in our list of 1000 random manifolds.

\subsection{Homology of particular representations}

As discussed in Section 3, if we look at a cover with covering group $Q$, the homology of the cover decomposes into

$$
H_{1}\left(G, V_{1}\right)^{n_{1}} \oplus H_{1}\left(G, V_{2}\right)^{n_{2}} \oplus \cdots \oplus H_{1}\left(G, V_{k}\right)^{n_{k}},
$$




$\begin{array}{rrrr}\text { Partition } & \text { Dim. of rep } & \text { Success rate } & \text { Mean homology } \\ 7 & 1 & 2 \% & 0.0 \\ 1,6 & 6 & 22 \% & 1.5 \\ 2,5 & 14 & 63 \% & 19.8 \\ 1,1,5 & 15 & 64 \% & 21.8 \\ 3,4 & 14 & 41 \% & 11.0 \\ 1,2,4 & 35 & 70 \% & 101.6 \\ 1,1,1,4 & 20 & 61 \% & 20.7 \\ 1,3,3 & 21 & 61 \% & 33.9\end{array}$

Table 3: The $\mathbb{Q}$-irreducible representations of $A_{7}$. Success Rate is the percentage of covers where that representation appeared. Mean Homology is the average amount of homology that that representation contributed (the mean homology of an $A_{7}$ cover was 210.3).

where $G$ is the fundamental group of the base manifold and the $V_{i}$ are the irreducible $Q$-modules. For $Q$ an alternating group, we looked at this decomposition and found that the ranks of the $H_{1}\left(G, V_{i}\right)$ were very strongly positively correlated. This contrasts with the relative independence of the ranks of covers with different $Q(n)$.

We will describe what happens for $A_{7}$, the other alternating groups being similar. The rational representations of $A_{7}$ are easy to describe: they are the restrictions of the irreducible representations of $S_{7}$. They correspond to certain partitions of 7 . Table 3 lists the representations and their basic properties. Table 4 shows the correlations between the ranks of the $H_{1}\left(G, V_{i}\right)$. Many of the correlations are larger than 0.5 and all are bigger than $0(+1$ is perfect correlation, -1 perfect anti-correlation and 0 the expected correlation for independent random variables). Figure 7 shows the distribution of the homology of the covers.

\subsection{Correlations between groups}

In the beginning of Section 5 we saw that the two events

(having a $Q(n)$-cover with $\beta_{1}>0$, having a $Q(m)$-cover with $\beta_{1}>0$ )

were more or less independent of each other, though overall there was a slight positive correlation which dampened the growth of $V(n)$. In the appendix, there is a table giving these correlations, was well one giving those between the events:

(having a $Q(n)$-cover, having a $Q(m)$-cover). 


\begin{tabular}{r|cccccccc} 
& 7 & 16 & 25 & 115 & 34 & 124 & 1114 & 133 \\
\hline 7 & $\mathbf{1 . 0 0}$ & 0.01 & 0.11 & 0.08 & 0.15 & 0.17 & 0.02 & 0.13 \\
16 & 0.01 & $\mathbf{1 . 0 0}$ & 0.22 & 0.09 & 0.23 & 0.19 & 0.18 & 0.19 \\
25 & 0.11 & 0.22 & $\mathbf{1 . 0 0}$ & 0.63 & 0.65 & 0.79 & 0.37 & 0.61 \\
115 & 0.08 & 0.09 & 0.63 & $\mathbf{1 . 0 0}$ & 0.52 & 0.80 & 0.75 & 0.78 \\
34 & 0.15 & 0.23 & 0.65 & 0.52 & $\mathbf{1 . 0 0}$ & 0.73 & 0.50 & 0.65 \\
124 & 0.17 & 0.19 & 0.79 & 0.80 & 0.73 & $\mathbf{1 . 0 0}$ & 0.65 & 0.89 \\
1114 & 0.02 & 0.18 & 0.37 & 0.75 & 0.50 & 0.65 & $\mathbf{1 . 0 0}$ & 0.66 \\
133 & 0.13 & 0.19 & 0.61 & 0.78 & 0.65 & 0.89 & 0.66 & $\mathbf{1 . 0 0}$
\end{tabular}

Table 4: Table showing the correlations between the ranks of $H_{1}\left(G, V_{i}\right)$ where the $V_{i}$ are indexed by the partition of the corresponding representation.

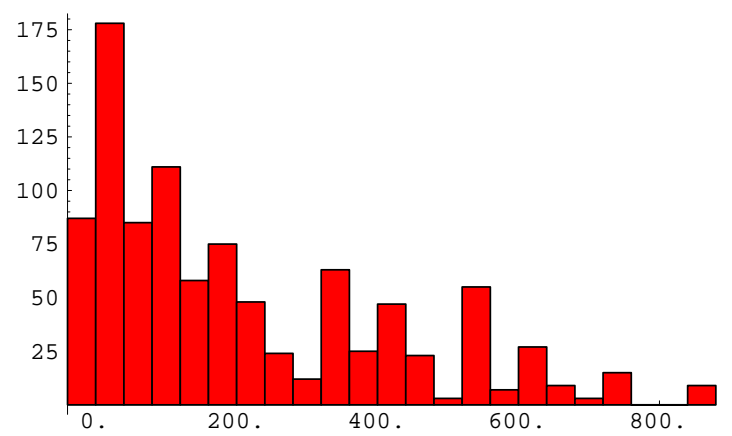

Figure 7: Plot showing the distribution of the ranks of the homology of the 964 covers with group $A_{7}$. The $x$-axis is the amount of homology and the $y$-axis the number of covers with homology in that range.

Some of these correlations are much larger than one would expect by chance alone - for instance the correlation between

( having a $L_{2}(7)$-cover with $\beta_{1}>0$, having a $L_{2}(8)$-cover with $\beta_{1}>0$ )

is 0.38 . Moreover, there are very few negative correlations and those that exist are quite small. Overall, the average correlation is positive as we would expect from Section 5.

One way of trying to understand these correlations is to observe that almost all of these manifolds are Dehn surgeries on the minimally twisted 5-chain. Let us focus on the simpler question of correlations between having a cover with group $Q(n)$ and having a cover with group $Q(m)$. Let $M$ be the complement of the 5 -chain. Consider all the homomorphisms $f_{k}: \pi_{1} M \rightarrow Q(n)$. Supposes 
$X$ is a Dehn filling on $M$ along the five slopes $\left(\gamma_{1}, \gamma_{2}, \gamma_{3}, \gamma_{4}, \gamma_{5}\right)$ where $\gamma_{i}$ is in $\pi_{1}\left(\partial_{i} M\right)$. The manifold $X$ has a cover with group $Q(n)$ if and only if there is an $f_{k}$ where each $\gamma_{i}$ lies in the kernel of $f_{k}$ restricted to $\pi_{1}\left(\partial_{i} M\right)$. Thus having a cover with group $Q(n)$ is determined by certain subgroups of the groups $\pi_{1}\left(\partial_{i} M\right)=\mathbb{Z}^{2}$. If we consider a different group $Q(m)$ we get a different family of subgroups of the $\pi_{1}\left(\partial_{i} M\right)$. If there is a lot of overlap between these two sets of subgroups, there will be a positive correlation between having a cover with group $Q(n)$ and having a cover with group $Q(m)$. If there is little overlap then there will be a negative correlation. However, even looked at this way there seems to be no reason that the average correlation should be positive.

If we look at the same question for manifolds which are Dehn surgeries on the figure- 8 knot (a simplified version of this setup) there are many negative correlations and the overall average correlation is 0 . If we look at the question for small surgeries on the Whitehead link, the overall average correlation is positive and of similar magnitude of that for the 5-chain. If we also look at larger surgeries on the Whitehead link the average correlation drops somewhat. By changing the link we get a different pattern of correlations, and so it is unwise to attach much significance to these numbers.

\section{$6 \quad$ Further questions}

Here are some interesting further questions related to our experiment.

(1) What happens for 3-manifolds bigger than the ones we looked at? Do the patterns we found persist? It is computationally difficult to deal with groups with large numbers of generators, which would limit the maximum size of the manifolds considered. But another difficulty is how to find a "representative" collection of such manifolds. (Some notions of a "random 3-manifold", which help with this latter question, will be discussed in [21]).

(2) How else could the virtually Haken covers we found be used to give insight into these conjectures? For instance, one could try to look at the virtual fibration conjecture. While there is no good algorithm for showing that a closed manifold is fibered, one could look at the following algebraic stand-in for this question. If a 3-manifold fibers over the circle, then one of the coefficients of the Alexander polynomial which is on a vertex of the Newton polytope is \pm 1 (see e.g. [18]). One could compute the Alexander polynomial of the covers with virtual positive betti number and see how 
often this occurred. As many of our covers are quite small, computing the Alexander polynomial should be feasible in many cases.

(3) One could use our methods to look at the Virtual Positive Betti Number conjecture for lattices in the other rank-1 groups that don't have Property T. This would be particularly interesting for the examples of complex hyperbolic manifolds where every congruence cover has $\beta_{1}=0$. These complex hyperbolic manifolds were discovered by Rogawski [47, Thm. 15.3.1] and are arithmetic.

\section{Transferring virtual Haken via Dehn filling}

In the rest of this paper, we consider the following setup. Let $M$ be a compact 3 -manifold with boundary a torus. The process of Dehn filling creates closed 3-manifolds from $M$ by taking a solid torus $D^{2} \times S^{1}$ and gluing its boundary to the boundary of $M$. The resulting manifolds are parameterized by the isotopy class of essential simple closed curve in $\partial M$ which bounds a disc in the attached solid torus. If $\alpha$ denotes such a class, called a slope, the corresponding Dehn filling is denoted by $M(\alpha)$. Though no orientation of $\alpha$ is needed for Dehn filling, we will often think of the possible $\alpha$ as being the primitive elements in $H_{1}(\partial M, \mathbb{Z})$ and so $H_{1}(\partial M, \mathbb{Z})$ parameterizes the possible Dehn fillings.

If you have a general conjecture which you can't prove for all 3-manifolds, a standard thing to do is to try to prove it for most Dehn fillings on an arbitrary 3-manifold with torus boundary. For instance, in the case of the Geometrization Conjecture there is the following theorem:

7.1 Hyperbolic Dehn Surgery Theorem [53] Let $M$ be a compact 3manifold with $\partial M$ a torus. Suppose the interior of $M$ has a complete hyperbolic metric of finite volume. Then all but finitely many Dehn fillings of $M$ are hyperbolic manifolds.

For the Virtual Haken Conjecture there is the following result of Cooper and Long. A properly embedded compact surface $S$ in $M$ is essential if it is incompressible, boundary incompressible, and not boundary parallel. Suppose $S$ is an essential surface in $M$. While $S$ may have several boundary components, they are all parallel and so have the same slope, called the boundary slope of $S$. If $\alpha$ and $\beta$ are two slopes, we denote their minimal intersection number, or distance, by $\Delta(\alpha, \beta)$. 
7.2 Theorem (Cooper-Long [12]) Let $M$ be a compact orientable 3-manifold with torus boundary which is hyperbolic. Suppose $S$ is a non-separating orientable essential surface in $M$ with non-empty boundary. Suppose that $S$ is not the fiber in a fibration over $S^{1}$. Let $\lambda$ be the boundary slope of $S$. Then there is a constant $N$ such that for all slopes $\alpha$ with $\Delta(\alpha, \lambda) \geq N$, the manifold $M(\alpha)$ is virtually Haken.

Explicitly, $N=12 g-8+4 b$ where $g$ is the genus of $S$ and $b$ is the number of boundary components.

This result differs from the Hyperbolic Dehn Surgery Theorem in that it excludes those fillings lying in an infinite strip in $H_{1}(\partial M)$, instead of only excluding those in a compact set. Here, we will prove a Dehn surgery theorem about the Virtual Positive Betti Number Conjecture, assuming that $M$ has a very simple Dehn filling which strongly has virtual positive betti number. Our theorem is a generalization of the work of Boyer and Zhang [5], which we discuss below.

The basic idea is this. Suppose $M$ has a Dehn filling $M(\alpha)$ which has virtual betti number in a very strong way. By this we mean that there is a surjection $\pi_{1}(M(\alpha)) \rightarrow \Gamma$ where $\Gamma$ is a group all of whose finite index subgroups have lots of homology. In our application, $\Gamma$ will be the fundamental group of a hyperbolic 2-orbifold. Given some other Dehn filling $M(\beta)$, we would like to transfer virtual positive betti number from $M(\alpha)$ to $M(\beta)$. Look at $\pi_{1}(M) /\langle\alpha, \beta\rangle$ which we will call $\pi_{1}(M(\alpha, \beta))$. This group is a common quotient of $\pi_{1}(M(\alpha))$ and $\pi_{1}(M(\beta))$. Choose $\gamma \in \pi_{1}(\partial M)$ so that $\{\alpha, \gamma\}$ is a basis of $\pi_{1}(\partial M)$. Then $\beta=\alpha^{m} \gamma^{n}$. If we think of $\pi_{1}(M(\alpha, \beta))$ as a quotient of $\pi_{1}(M(\alpha))$ we have:

$$
\pi_{1}(M(\alpha, \beta))=\pi_{1}(M(\alpha)) /\langle\beta\rangle=\pi_{1}(M(\alpha)) /\left\langle\gamma^{n}\right\rangle .
$$

Thus $\pi_{1}(M(\alpha, \beta))$ surjects onto $\Gamma /\left\langle\gamma^{n}\right\rangle$, where here we are confusing $\gamma$ and its image in $\Gamma$. So $\pi_{1}(M)$ surjects onto $\Gamma /\left\langle\gamma^{n}\right\rangle$. If $\Gamma$ has rapid homology growth, one can hope that $\Gamma_{n}=\Gamma /\left\langle\gamma^{n}\right\rangle$ still has virtual positive betti number when $n$ is large enough. This is plausible because adding a relator which is a large power often doesn't change the group too much. If there is an $N$ so that $\Gamma_{n}$ has virtual positive betti number for all $n \geq N$, then $M(\beta)$ has virtual positive betti number for all $\beta$ with $n=\Delta(\gamma, \alpha) \geq N$.

Our main theorem applies when $M(\alpha)$ is a Seifert fibered space whose base orbifold is hyperbolic:

7.3 Theorem Let $M$ be a compact 3-manifold with boundary a torus. Suppose $M(\alpha)$ is Seifert fibered with base orbifold $\Sigma$ hyperbolic. Assume also 
that the image of $\pi_{1}(\partial M)$ under the induced map $\pi_{1}(M) \rightarrow \pi_{1}(\Sigma)$ contains no non-trivial element of finite order. Then there exists an $N$ so that $M(\beta)$ has virtual positive betti number whenever $\Delta(\alpha, \beta) \geq N$.

If $\Sigma$ is not a sphere with 3 cone points, then $N$ can be taken to be 7 .

In light of the above discussion, if we consider the homomorphism $\pi_{1}(M(\alpha)) \rightarrow$ $\pi_{1}(\Sigma)=\Gamma$, Theorem 7.3 follows immediately from:

7.4 Theorem Let $\Sigma$ be a closed hyperbolic 2-orbifold without mirrors, and $\Gamma$ be its fundamental group. Let $\gamma \in \Gamma$ be a element of infinite order. Then there exists an $N$ such that for all $n \geq N$ the group

$$
\Gamma_{n}=\Gamma /\left\langle\gamma^{n}\right\rangle
$$

has virtual positive betti number. In fact, $\Gamma_{n}$ has a finite index subgroup which surjects onto a free group of rank 2.

If $\Sigma$ is not a 2 -sphere with 3 cone points, then $N=\max \{1 /|1+\chi(\Sigma)|, 3\}$. In this case, $N$ is at most 7 .

In applying Theorem 7.3, the technical condition that the image of $\pi_{1}(\partial M)$ not contain an element of finite order holds in many cases. For instance, Theorem 7.3 implies the following theorem about Dehn surgeries on the Whitehead link. Let $W$ the exterior of the Whitehead link. Given a slope $\alpha$ on the first boundary component of $W$, we denote by $W(\alpha)$ the manifold with one torus boundary component obtained by filling along $\alpha$.

Theorem (9.1) Let $W$ be the exterior of the Whitehead link. Then for all but finitely many slopes $\alpha$, the manifold $M=W(\alpha)$ has the following property: All but finitely many Dehn fillings of $M$ have virtual positive betti number.

In fact, our proof of this theorem excludes only 28 possible slopes $\alpha$ (see Section 9). The complements of the twist knots in $S^{3}$ are exactly the $W(1 / n)$ for $n \in \mathbb{Z}$. Theorem 9.1 applies to all of the slopes $1 / n$ except for $n \in\{0,1\}$ which correspond to the unknot and the trefoil. Thus we have:

7.5 Corollary Let $K$ be a twist knot in $S^{3}$ which is not the unknot or the trefoil. Then all but finitely many Dehn surgeries on $K$ have virtual positive betti number.

For the simplest hyperbolic knot, the figure-8, we can use a quantitative version of Theorem 7.4 due to Holt and Plesken [35] which applies in this special case. We will show: 
7.6 Theorem Every non-trivial Dehn surgery on the figure-8 knot in $S^{3}$ has virtual positive betti number.

As we mentioned, Theorem 7.3 generalizes the work of Boyer and Zhang [5]. They restricted to the case where the base orbifold was not a 2 -sphere with 3 cone points. In particular, they proved:

7.7 Theorem [5] Let $M$ have boundary a torus. Suppose $M(\alpha)$ is Seifert fibered with a hyperbolic base orbifold $\Sigma$ which is not a 2-sphere with 3 cone points. Assume also that $M$ is small, that is, contains no closed essential surface. Then $M(\beta)$ has virtual positive betti number whenever $\Delta(\alpha, \beta) \geq 7$.

The condition that $M$ is small is a natural one as if $M$ contains an closed essential surface, then there is a $\alpha$ so that $M(\beta)$ is actually Haken if $\Delta(\alpha, \beta)>1$ $[15,57]$.

Boyer and Zhang's point of view is different than ours, in that they do not set out a restricted version of Theorem 7.4. While the basic approach of both proofs comes from [2], Boyer and Zhang's proof of Theorem 7.7 also uses the Culler-Shalen theory of $\mathrm{SL}_{2} \mathbb{C}$-character varieties and surfaces arising from ideal points. From our point of view this is not needed, and Theorem 7.7 follows easily from Theorem 7.3 (see the end of Section 8 for a proof).

In Section 11, we discuss possible generalizations of Theorem 7.3 to other types of fillings. In a very special case, we use toroidal Dehn fillings to show (Theorem 12.1) that every Dehn filling of the sister of the figure-8 complement satisfies the Virtual Positive Betti Number Conjecture.

\section{One-relator quotients of 2-orbifold groups}

This section is devoted to the proof of Theorem 7.4. The basic ideas go back to $[2]$ which proves the analogous result for $\Gamma=\mathbb{Z} / p * \mathbb{Z} / q$. Fine, Roehl, and Rosenberger proved Theorem 7.4 in many, but not all, cases where $\Sigma$ is not a 2 -sphere with 3 cone points $[22,23]$. In the case $\Sigma=S^{2}\left(a_{1}, a_{2}, a_{3}\right)$, Darren Long and Alan Reid suggested the proof given below, and Matt Baker provided invaluable help with the number theoretic details.

Proof of Theorem 7.4 Let $\Sigma_{n}$ be the 2-complex with marked cone points consisting of $\Sigma$ together with a disc $D$ with a cone point of order $n$, where the boundary of $D$ is attached to $\Sigma$ along a curve representing $\gamma$. Thus $\Gamma_{n}=$ 
$\pi_{1}\left(\Sigma_{n}\right)$. Now the Euler characteristic of $\Sigma_{n}$ is $\chi(\Sigma)+1 / n$, which is negative if $n>1 /|\chi(\Sigma)|$. From now on, assume that $n>1 /|\chi(\Sigma)|$. Suppose $\Gamma_{n}$ contains a subgroup $\Gamma_{n}^{\prime}$ of finite index such that if $\alpha$ is a small loop about a cone point then $\alpha \notin \Gamma_{n}^{\prime}$. For instance, this is the case if $\Gamma_{n}^{\prime}$ is torsion free. Let $\Sigma_{n}^{\prime}$ be the corresponding cover of $\Sigma_{n}$, so $\Gamma_{n}^{\prime}=\pi_{1}\left(\Sigma_{n}^{\prime}\right)$. Then $\Sigma_{n}^{\prime}$ is a 2-complex without any cone points. Since $\Sigma_{n}^{\prime}$ has negative Euler characteristic and there is no homology in dimensions greater than two, we must have $H_{1}\left(\Sigma_{n}^{\prime}, \mathbb{Q}\right) \neq 0$. Thus $\Gamma_{n}$ has virtual positive betti number.

One can show more: Let $d$ be the degree of the cover $\Sigma_{n}^{\prime} \rightarrow \Sigma_{n}$. The complex $\Sigma_{n}^{\prime}$ is a smooth hyperbolic surface $S$ with $d / n$ discs attached. From this description it is easy to check that $\Gamma_{n}^{\prime}$ has a presentation where

$$
\begin{aligned}
(\# \text { of generators })-(\# \text { of relations }) & =(|\chi(S)|+1)-\frac{d}{n} \\
& =1+d\left(|\chi(\Sigma)|-\frac{1}{n}\right) \geq 2 .
\end{aligned}
$$

By a theorem of Baumslag and Pride [1], the group $\Gamma_{n}^{\prime}$ has a finite-index subgroup which surjects onto $\mathbb{Z} * \mathbb{Z}$.

So it remains to produce the subgroups $\Gamma_{n}^{\prime}$. First, we discuss the case where $\Sigma$ is not a sphere with 3 cone points. A homomorphism $f: \Gamma \rightarrow Q$ is said to preserve torsion if for every torsion element $\alpha$ in $\Gamma$ the order of $f(\alpha)$ is equal to the order of $\alpha$. (Recall that the torsion elements of $\Gamma$ are exactly the loops around cone points.) The key is to show:

8.1 Lemma Suppose $\Sigma$ is not a 2-sphere with 3 cone points, and that $\gamma \in \Gamma$ has infinite order. Given any $n>2$, there exists a homomorphism $\rho: \Gamma \rightarrow$ $\mathrm{PSL}_{2} \mathbb{C}$ such that $\rho$ preserves torsion and $\rho(\gamma)$ has order $n$.

Suppose we have $\rho$ as in the lemma, which we will regard as a homomorphism from $\Gamma_{n}$ to $\mathrm{PSL}_{2} \mathbb{C}$. By Selberg's lemma, the group $\rho(\Gamma)$ has a finite index subgroup $\Lambda$ which is torsion free. We can then take $\Gamma_{n}^{\prime}$ to be $\rho^{-1}(\Lambda)$. Because the lemma only requires that $n>2$ and the preceding argument required that $n>1 /|\chi(\Sigma)|$, in this case we can take the $N$ in the statement of Theorem 7.4 to be $\max \{3,1+1 /|\chi(\Sigma)|\}$. A case check, done in [5], shows that $N$ is at most 7. As we will see, the proof of Lemma 8.1 is relatively easy and involves deforming Fuchsian representations $\Gamma \rightarrow \operatorname{Isom}\left(\mathbb{H}^{2}\right)$ to find $\rho$.

The harder case is when $\Sigma$ is a 2 -sphere with 3 cone points, which we denote $S^{2}\left(a_{1}, a_{2}, a_{3}\right)$. Here the fundamental group $\Gamma$ can be presented as

$$
\left\langle x_{1}, x_{2}, x_{3} \mid x_{1}^{a_{1}}=x_{2}^{a_{2}}=x_{3}^{a_{3}}=x_{1} x_{2} x_{3}=1\right\rangle .
$$


Geometrically, $x_{i}$ is a loop around the $i^{\text {th }}$ cone point. We will show:

8.2 Lemma Let $\Gamma=\pi_{1}\left(S^{2}\left(a_{1}, a_{2}, a_{3}\right)\right)$ where $1 / a_{1}+1 / a_{2}+1 / a_{3}<1$. Given an element $\gamma \in \Gamma$ of infinite order, there exists an $N$ such that for all $n \geq N$ the group $\Gamma$ has a finite quotient where the images of $\left(x_{1}, x_{2}, x_{3}, \gamma\right)$ have orders exactly $\left(a_{1}, a_{2}, a_{3}, n\right)$ respectively.

With this Lemma, we can take $\Gamma_{n}^{\prime}$ to be the kernel of the given finite quotient. The proof of Lemma 8.2 involves using congruence quotients of $\Gamma$ and a some number theory. Unfortunately, unlike the previous case, the proof of Lemma 8.2 gives no explicit bound on $N$.

In any event, we've established Theorem 7.4 modulo Lemmas 8.1 and 8.2.

The rest of this section is devoted to proving the two lemmas.

Proof of Lemma 8.1 Because $\Sigma$ is not a 2-sphere with 3 cone points, the Teichmüller space of $\Sigma$ is positive dimensional. Thus there are many representations of $\Gamma$ into Isom $\left(\mathbb{H}^{2}\right)$. We can embed Isom $\left(\mathbb{H}^{2}\right)$ into $\operatorname{Isom}^{+}\left(\mathbb{H}^{3}\right)=\mathrm{PSL}_{2} \mathbb{C}$ as the stabilizer of a geodesic plane. We will then deform these Fuchsian representations to produce $\rho$.

Pick a simple closed curve $\beta$ which intersects $\gamma$ essentially. There are two cases depending on whether a neighborhood of $\beta$ is an annulus or a Möbius band.

Suppose the neighborhood is an annulus. First, let's consider the case where $\beta$ separates $\Sigma$ into 2 pieces. In this case $\Gamma$ is a free product with amalgamation $A *\langle\beta\rangle$. Let $\rho_{1}: \Gamma \rightarrow \mathrm{PSL}_{2} \mathbb{C}$ be one of the Fuchsian representations. Conjugate $\rho_{1}$ so that $\rho_{1}(\beta)$ is diagonal. Then $\rho_{1}(\beta)$ commutes with the matrices

$$
C_{t}=\left(\begin{array}{cc}
t & 0 \\
0 & t^{-1}
\end{array}\right) \text { for } t \text { in } \mathbb{C}^{\times} .
$$

For $t$ in $\mathbb{C}^{\times}$, let $\rho_{t}$ be the representation of $\Gamma$ whose restriction to $A$ is $\rho_{1}$ and whose restriction to $B$ is $C_{t} \rho_{1} C_{t}^{-1}$. Consider the function $f: \mathbb{C}^{\times} \rightarrow \mathbb{C}$ which sends $t$ to $\operatorname{tr}^{2}\left(\rho_{t}(\gamma)\right)$. It is easy to see that $f$ is a rational function of $t$ by expressing $\gamma$ as a word in elements of $A$ and $B$. We claim that $f$ is nonconstant. First, suppose that neither of the two components of $\Sigma \backslash \beta$ is a disc with two cone points of order 2 . In this case, $\beta$ can be taken to be a geodesic loop. If we restrict $t$ to $\mathbb{R}$ then the family $\left\{\rho_{t}\right\}$ corresponds to twisting around $\beta$ in the Fenchel-Nielsen coordinates on Teich $(\Sigma)$. As $\gamma$ intersects $\beta$ essentially, the length of $\gamma$ changes under this twisting and so $f$ is non-constant. From 
this same point of view, we see that that $f$ has poles at 0 and $\infty$. If one of the pieces of $\Sigma \backslash \beta$ is a disc with two cone points of order 2 , then $\beta$ naturally shrinks not to a closed geodesic, but to a geodesic arc joining the two cone points. There is still a Fenchel-Nielsen twist about $\beta$, and so we have the same observations about $f$ in this case (think of $\Sigma$ being obtained from a surface with a geodesic boundary component by pinching the boundary to a interval).

Since the rational function $f$ has poles at $\{0, \infty\}$, we have $f\left(\mathbb{C}^{\times}\right)=\mathbb{C}$. So given $n>1$, we can choose $t \in \mathbb{C}^{\times}$so that $\operatorname{tr}^{2}\left(\rho_{t}(\gamma)\right)=\left(\zeta_{2 n}+\zeta_{2 n}^{-1}\right)^{2}$ where $\zeta_{2 n}=e^{\pi i / n}$. Then $\rho_{t}(\gamma)$ has order $n$. Moreover, $\rho_{t}$ preserves torsion because $\rho_{1}$ does, and so we have finished the proof of the lemma when $\beta$ is separating and has an annulus neighborhood. If $\beta$ has an annulus neighborhood and is nonseparating, the proof is identical except that $\Gamma$ is an HNN-extension instead of a free product with amalgamation.

Now we consider the case where the neighborhood of $\beta$ is a Möbius band. The difference here is that you can't twist a hyperbolic structure of $\Sigma$ along $\beta$. To see this, think of constructing $\Sigma$ from a surface with geodesic boundary where the boundary is identified by the antipodal map to form $\beta$. Instead, we will deform the length of $\beta$ in Teich $(\Sigma)$. Here we will need the hypothesis that $n>2$, as you can see by looking at $\mathbb{R} \mathrm{P}^{2}(3,5)$ with $\gamma$ a simple closed geodesic which has a Möbius band neighborhood. The only quotient of $\pi_{1}\left(\mathbb{R} \mathrm{P}^{2}(3,5)\right)$ where $\gamma$ has order 2 is $\mathbb{Z} / 2$ and this doesn't preserve torsion.

The underlying surface of $\Sigma$ is non-orientable. We can assume that $\Sigma$ has at least one cone point since every non-orientable surface covers such an orbifold. Pick an arc $a$ joining $\beta$ to a cone point $p$. Let $A$ be a closed neighborhood of $\beta \cup a$. The set $A$ is a Möbius band with a cone point. Let $B$ be the closure of $\Sigma \backslash A$. Let $\alpha$ be the boundary of $A$. A small neighborhood of $\alpha$ is an annulus, so if $\gamma$ intersects $\alpha$ essentially, we can replace $\beta$ with $\alpha$ and use the argument above. So from now on, we can assume that $\gamma$ lies in $A$. Let $\psi: \Gamma \rightarrow \mathrm{PSL}_{2} \mathbb{C}$ be a Fuchsian representation. Suppose we construct a representation $\rho: \pi_{1}(A) \rightarrow \mathrm{PSL}_{2} \mathbb{C}$ so that $\rho$ preserves torsion, $\rho(\gamma)$ has order $n$, and $\operatorname{tr}^{2}(\rho(\alpha))=\operatorname{tr}^{2}(\psi(\alpha))$. Then as $\Gamma=\pi_{1}(A) *\langle\alpha\rangle \pi_{1}(B)$ and $\rho$ and $\psi$ are conjugate on $\langle\alpha\rangle$, we can glue $\rho$ and $\psi$ restricted to $\pi_{1}(B)$ together to get the required representation of $\Gamma$.

Thus we have reduced everything to a question about certain representations of $\pi_{1}(A)$. The group $\pi_{1}(A)$ is generated by $\alpha$ and $\beta$. Choosing orientations correctly, a small loop about the cone point $p$ is $\delta=\beta^{2} \alpha$. If $p$ has order $r$, then $\pi_{1}(A)$ has the presentation

$$
\left\langle\alpha, \beta, \delta \mid \delta=\beta^{2} \alpha, \delta^{r}=1\right\rangle .
$$


Given any representation $\phi$ of $\pi_{1}(A)$, we will fix lifts of $\phi(\alpha)$ and $\phi(\beta)$ to $\mathrm{SL}_{2} \mathbb{C}$. Having done this, any word $w$ in $\alpha$ and $\beta$ has a canonical lift of $\phi(w)$ to $\mathrm{SL}_{2} \mathbb{C}$. We will abuse notion and denote this lift by $\phi(w)$ as well. In this way, we can treat $\phi$ as though it was a representation into $\mathrm{SL}_{2} \mathbb{C}$ so that, for instance, the trace of $\phi(w)$ is defined.

Define a 1-parameter family of representations $\rho_{t}$ for $t \in \mathbb{C}^{\times}$as follows. Set

$$
\psi(\beta)=\left(\begin{array}{cc}
0 & 1 \\
-1 & t
\end{array}\right), \quad \text { and } \quad \psi(\alpha)=\left(\begin{array}{cc}
e & s \\
0 & e^{-1}
\end{array}\right)
$$

where $e+e^{-1}=\operatorname{tr}(\psi(\alpha))$ and $s=\frac{1}{t}\left(e^{-1} t^{2}-\left(e+e^{-1}\right)-\operatorname{tr}(\psi(\delta))\right.$. This gives a representation of $\pi_{1}(A)$ because $s$ was chosen so that $\operatorname{tr}\left(\rho_{t}(\delta)\right)=\operatorname{tr}(\psi(\delta))$ and so $\rho_{t}(\delta)$ also has order $r$ in $\mathrm{PSL}_{2} \mathbb{C}$.

Let Teich $(A)$ denote hyperbolic structures on $A$ with geodesic boundary where the length of the boundary is fixed to be that of the Fuchsian representation $\psi$. This Teichmüller space is $\mathbb{R}$ with the single Fenchel-Nielsen coordinate being the length of $\beta$. Note that any irreducible representation of $\pi_{1}(A)$ is conjugate to some $\rho_{t}$, and so each point in Teich $(A)$ yields a Fuchsian representation $\rho_{t}$. As $\beta$ gets short in Teich $(A)$, the curve $\gamma$ gets long. Thus if we set $f=\operatorname{tr}\left(\rho_{t}(\gamma)\right)$, then $f$ is a non-constant Laurent polynomial in $t$.

Let $v=\zeta_{2 n}+\zeta_{2 n}^{-1}$. To finish the proof of the lemma, all we need to do is find a $t \in \mathbb{C}^{\times}$so that $f(t)^{2}=v^{2}$. As a map from the Riemann sphere to itself, $f$ is onto and there are $t_{1}$ and $t_{2}$ in $\widehat{\mathbb{C}}$ so that $f\left(t_{1}\right)=v$ and $f\left(t_{2}\right)=-v$. As $n>2, v$ is not 0 and so $t_{1}$ and $t_{2}$ are distinct. As $f$ is non-constant and finite on $\mathbb{C}^{\times}$, it has a pole at at least one of 0 and $\infty$. Therefore, at least one of $t_{1}$ and $t_{2}$ is in $\mathbb{C}^{\times}$and we are done.

Proof of Lemma 8.2 The group $\Gamma$ is naturally a subgroup of $\mathrm{PSL}_{2} \mathbb{R}$. Set $b_{i}=2 a_{i}$. Let $X_{i}$ be the matrix in $\mathrm{PSL}_{2} \mathbb{R}$ corresponding to the generator $x_{i}$. As $X_{i}$ has order $a_{i}$, it follows that $\operatorname{tr}\left(X_{i}\right)= \pm\left(\zeta_{b_{i}}+\zeta_{b_{i}}^{-1}\right)$ where $\zeta_{b_{i}}$ is some primitive $b_{i}{ }^{\text {th }}$ root of unity. Any irreducible 2-generator subgroup of $\mathrm{PSL}_{2} \mathbb{C}$ is determined by its traces on the generators and their product, and so we can conjugate $\Gamma$ in $\mathrm{PSL}_{2} \mathbb{C}$ so the $X_{i}$ are:

$$
X_{1}=\left(\begin{array}{cc}
0 & 1 \\
-1 & \zeta_{b_{1}}+\zeta_{b_{1}}^{-1}
\end{array}\right), X_{2}=\left(\begin{array}{cc}
\zeta_{b_{2}}+\zeta_{b_{2}}^{-1} & -\zeta_{b_{3}} \\
\zeta_{b_{3}}^{-1} & 0
\end{array}\right) \text {, and } X_{3}=\left(X_{1} X_{2}\right)^{-1} \text {. }
$$

Henceforth we will identify $\Gamma$ with its image. The entries of the $X_{i}$ lie in $\mathbb{Q}\left(\zeta_{b_{1}}, \zeta_{b_{2}}, \zeta_{b_{3}}\right)$, and moreover are integral, so $\Gamma$ is contained in the subgroup $\mathrm{PSL}_{2} \mathcal{O}\left(\mathbb{Q}\left(\zeta_{b_{1}}, \zeta_{b_{2}}, \zeta_{b_{3}}\right)\right)$. Let $G$ be a matrix in $\mathrm{PSL}_{2} \mathbb{C}$ representing $\gamma$. Let $a$ 
be one of the eigenvalues of $G$. Note that $a$ is an algebraic integer, in fact a unit, because it satisfies the equation $a^{2}-(\operatorname{tr} G) a+1$ and $\operatorname{tr} G$ is integral. Let $K$ be the field $\mathbb{Q}\left(\zeta_{b_{1}}, \zeta_{b_{2}}, \zeta_{b_{3}}, a\right)$. From now on, we will consider $\Gamma$ as a subgroup of $\mathrm{PSL}_{2} \mathcal{O}(K)$. We will construct the required quotients of $\Gamma$ from congruence quotients of $\mathrm{PSL}_{2} \mathcal{O}(K)$. Suppose $\wp$ is a prime ideal of $\mathcal{O}(K)$. Setting $k=\mathcal{O}(K) / \wp$, we have the finite quotient of $\Gamma$ given by

$$
\Gamma \rightarrow \mathrm{PSL}_{2} \mathcal{O}(K) \rightarrow \mathrm{PSL}_{2} k .
$$

What conditions do we need so that $\left(x_{1}, x_{2}, x_{3}, \gamma\right)$ have the right orders in $\mathrm{PSL}_{2} k$ ? Well, the eigenvalues of $X_{i}$ are $\pm\left\{\zeta_{b_{i}}, \zeta_{b_{i}}^{-1}\right\}$, so as long as $\bar{\zeta}_{b_{i}}$ has order $b_{i}$ in $k^{\times}$, the matrix $\bar{X}_{i}$ in $\mathrm{PSL}_{2} k$ also has order $b_{i}$. Similarly, if we set $m=2 n$, then $\bar{G}$ in $\mathrm{PSL}_{2} k$ has order $n$ if $\bar{a}$ has order $m$ in $k^{\times}$. Thus the following claim will complete the proof of the lemma:

8.3 Claim There exists an $N$ such that for all $n \geq N$ there is a prime ideal $\wp$ such that if $k=\mathcal{O}(K) / \wp$ then the images of $\left(\zeta_{b_{1}}, \zeta_{b_{2}}, \zeta_{b_{3}}, a\right)$ in $k^{\times}$have orders $\left(b_{1}, b_{2}, b_{3}, m\right)$.

Let's prove the claim. The idea is to show that $a^{m}-1$ is not a unit in $\mathcal{O}(K)$ for large $m$, and then just take $\wp$ to be a prime ideal dividing $a^{m}-1$. We have to be careful, though, that $\left(\bar{\zeta}_{b_{1}}, \bar{\zeta}_{b_{2}}, \bar{\zeta}_{b_{3}}, \bar{a}\right)$ don't end up with lower orders that expected in $k^{\times}$.

A prime ideal is called primitive if it divides $a^{m}-1$ and does not divide $a^{r}-1$ for all $r<m$. Postnikova and Schinzel proved the following theorem:

8.4 Theorem $[48,46]$ Suppose that $a$ is an algebraic integer which is not a root of unity. There there is an $N$ such that for all $n \geq N$ the integer $a^{n}-1$ has a primitive divisor.

The proof of Theorem 8.4 relies on deep theorems of Gelfond and A. Baker on the approximation by rationals of logarithms of algebraic numbers.

Because $\gamma$ has infinite order, we know that $a$ is not a root of unity. Thus Theorem 8.4 applies, and let $N$ be as in the statement. By increasing $N$ if necessary, we can ensure that the primitive divisor $\wp$ given Theorem 8.4 does not divide any element of the finite set

$$
R=\left\{\zeta_{b_{i}}^{r}-1 \mid 1 \leq r<b_{i}\right\} .
$$

Thus for all $m \geq N$, we have a prime ideal $\wp$ which divides $a^{m}-1$ but does not divide $a^{r}-1$ for $r<m$. Thus $\bar{a}$ has order $m$ in $k^{\times}$. As $\wp$ does not divide any element of $R$, the element $\bar{\zeta}_{b_{i}}$ has order $b_{i}$ in $k^{\times}$. This proves the claim and thus the lemma. 
It would be nice to have given a proof of Lemma 8.2 which gave an explicit bound on $N$. The number theory used gives "an effectively computable constant" for $N$, but doesn't actually compute it. Perhaps there are other proofs of Lemma 8.2 more like that of Lemma 8.1. While $\pi_{1}\left(S^{2}\left(a_{1}, a_{2}, a_{3}\right)\right)$ has only a finite number of representations into $\mathrm{PSL}_{2} \mathbb{C}$, if one looks at representations into larger groups there are deformation spaces where you could hope to play the same game. For instance, if one embeds $\mathbb{H}^{2}$ as a totally geodesic subspace in complex hyperbolic space $\mathbb{C} H^{2}$, then a Fuchsian representation deforms to a one real parameter family in $\operatorname{Isom}^{+}\left(\mathbb{C H}^{2}\right)=\mathrm{PU}(2,1)$. One could instead consider deformations in the space of real-projective structures, which gives rise to homomorphisms to $\mathrm{PGL}_{3} \mathbb{R}$ [10]. In general, the structure of the space representations of $\pi_{1}\left(S^{2}\left(a_{1}, a_{2}, a_{3}\right)\right) \rightarrow \mathrm{SL}_{n} \mathbb{C}$ is closely related to the Deligne-Simpson problem [51].

We end this section by deducing Boyer and Zhang's original Theorem 7.7 from Theorem 7.3.

Proof of Theorem 7.7 Let $M$ be a manifold with torus boundary which is small. Suppose that $M(\alpha)$ is Seifert fibered with hyperbolic base orbifold $\Sigma$ which is not sphere with 3 cone points. We need to check that Theorem 7.3 applies. Let $\beta$ be a curve so that $\{\alpha, \beta\}$ is a basis for $\pi_{1}(\partial M)$. It suffices to show the image of $\beta$ does not have finite order in $\Gamma=\pi_{1}(\Sigma)$. Suppose not. Then there are infinitely many Dehn fillings $M\left(\gamma_{i}\right)$ of $M$ where $\pi_{1}\left(M\left(\gamma_{i}\right)\right)$ surjects onto $\Gamma$. The orbifold $\Sigma$ contains an essential simple closed curve which isn't a loop around a cone point. Therefore, $\Gamma$ has non-trivial splitting as a graph of groups and so acts non-trivially on a simplicial tree. Then each $\pi_{1}\left(M\left(\gamma_{i}\right)\right)$ act non-trivially on a tree and so $M\left(\gamma_{i}\right)$ contains an essential surface. As infinitely many fillings contain essential surfaces, a theorem of Hatcher [30] implies that $M$ contains a closed essential surface. This is contradicts that $M$ is small. So the image of $\beta$ has infinite order and we are done.

\section{Surgeries on the Whitehead link}

Consider the Whitehead link pictured in Figure 8. Let $W$ be its exterior. We will denote the two boundary components of $W$ by $\partial_{0} W$ and $\partial_{1} W$. For each $\partial_{i} W$, we fix a meridian-longitude basis $\left\{\mu_{i}, \lambda_{i}\right\}$ with the orientations shown in the figure. With respect to one of these bases, we will write boundary slopes as rational numbers, where $p \mu+q \lambda$ corresponds to $p / q$. We will denote Dehn filling of both boundary components of $W$ by $W\left(p_{0} / q_{0} ; p_{1} / q_{1}\right)$. Dehn filling 


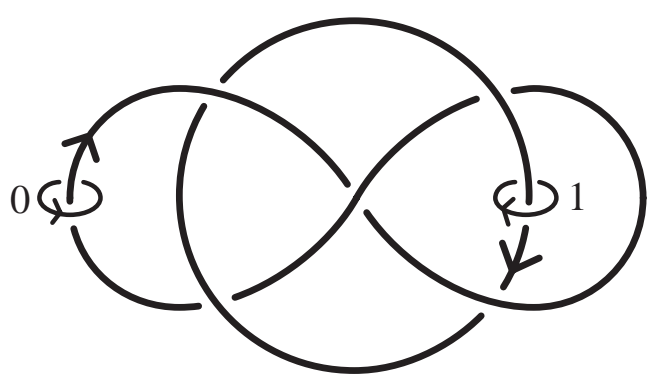

Figure 8: The Whitehead link, showing our orientation conventions for the meridians and longitudes.

on a single component of $W$ will be denoted $W\left(p_{0} / q_{0} ; \cdot\right)$ and $W\left(\cdot ; p_{1} / q_{1}\right)$. As $W(p / q ; \cdot)$ is homeomorphic to $W(\cdot ; p / q)$, we will sometimes denote this manifold by $W(p / q)$. With our conventions, $W(1)$ is the trefoil complement, and $W(-1)$ is the figure- 8 complement. The manifold $W(p / q)$ is hyperbolic except when $p / q$ is in $\{\infty, 0,1,2,3,4\}$. The point of this section is to show:

9.1 Theorem Let $W$ be the complement of the Whitehead link. For any slope $p / q$ which is not in $E=\{\infty, 0,1,2,3,4,5,5 / 2,6,7 / 1,7 / 2,8$, $8 / 3,9 / 2,10 / 3,11 / 2,11 / 3,13 / 3,13 / 4,14 / 3,15 / 4,16 / 3,16 / 5,17 / 5,18 / 5$, $19 / 4,24 / 5,24 / 7\}$ the manifold $W(\alpha)$ has the property that all but finitely many Dehn fillings have virtual positive betti number.

Proof The proof goes by showing that except for $p / q$ in $E$, the manifold $W(p / q)$ has at least 2 distinct Dehn fillings which are Seifert fibered and to which Theorem 7.3 applies. The reason that $W(p / q)$ has so many Seifert fibered fillings is because the manifolds $W(1), W(2)$, and $W(3)$ are all Seifert fibered with base orbifold a disc with two cone points. In particular, the base orbifolds are $D^{2}(2,3), D^{2}(2,4)$, and $D^{2}(3,3)$ respectively. Therefore, all but one Dehn surgery $W(1 ; p / q)$ on $W(1)$ is Seifert fibered with base orbifold a sphere with 3 cone points. Similarly for $W(2)$ and $W(3)$. In fact, you can check that

- $W(1 ; p / q)$ Seifert fibers over $S^{2}(2,3,|p-6 q|)$ if $p / q \neq 6$.

- $W(2 ; p / q)$ Seifert fibers over $S^{2}(2,4,|p-4 q|)$ if $p / q \neq 4$.

- $W(3 ; p / q)$ Seifert fibers over $S^{2}(3,3,|p-3 q|)$ if $p / q \neq 3$.

Now fix a slope $p / q$, and consider the manifold $M=W(\cdot ; p / q)$. We want to know when we can apply Theorem 7.3 to $M(1), M(2)$, or $M(3)$. First, we 
need the base orbifold to be hyperbolic, i.e. that the reciprocals of the orders of the cone points sum to less than 1 . This leads to the conditions:

$$
\begin{aligned}
& \text { For } M(1) \text { that }|p-6 q|>6 . \\
& \text { For } M(2) \text { that }|p-4 q|>4 . \\
& \text { For } M(3) \text { that }|p-3 q|>3 .
\end{aligned}
$$

We claim that as long as the base orbifold is hyperbolic then Theorem 7.3 applies. Consider the map $\pi_{1}(M) \rightarrow \Gamma$ where $\Gamma$ is the fundamental group of one of the base orbifolds. Let $\mu$ in $\partial M$ be the meridian coming from our meridian $\mu_{0}$ of $W$. Since $\mu$ intersects any of the slopes $1,2,3$ once, its image in $\Gamma$ generates the image of $\pi_{1}(\partial M)$. Thus we just need to check that the image of $\mu$ is an element of infinite order in $\Gamma$. One can work out what the image in $\Gamma$ is explicitly (most easily by with the help of SnapPea [56]):

For $M(1), \mu \mapsto a b a^{-1} b^{-1}$ where

$$
\Gamma=\left\langle a, b \mid a^{2}=b^{3}=(a b)^{p-6 q}=1\right\rangle .
$$

For $M(2), \mu \mapsto a b^{2}$ where

$$
\Gamma=\left\langle a, b \mid a^{2}=b^{4}=(a b)^{p-4 q}=1\right\rangle .
$$

For $M(3), \mu \mapsto a b^{-1}$ where

$$
\Gamma=\left\langle a, b \mid a^{3}=b^{3}=(a b)^{p-3 q}=1\right\rangle .
$$

It remains to check that the images of $\mu$ above always have infinite order in $\Gamma$. This is intuitively clear for looking at loops which represent these elements. The suspicious reader can check that this is really the case by using, say, the solution to the word problem for Coxeter groups [6, $\S$ II.3].

Thus, Theorem 7.3 applies whenever one of the conditions in (2) holds. If $p / q$ is such that two of (2) hold, then all but finitely many Dehn surgeries on $M$ have virtual positive betti number. The set in $H_{1}(\partial M, \mathbb{R})=\mathbb{R}^{2}$ where any one of the conditions fails is an infinite strip. So the set where a fixed pair of them fail is compact, namely a parallelogram. Hence, outside a union of 3 parallelograms, at least two of the conditions hold. These 3 parallelograms are all contained in the square where $|p|,|q| \leq 100$. To complete the proof of the theorem, one checks all the slopes in that square to find those where fewer that two of (2) hold.

For most of the slopes in $E$, one of (2) holds, and so one still has a partial result. The slopes where none of the conditions in (2) hold are

$$
\{\infty, 0,1,2,3,4,5,6,7 / 2,9 / 2\} .
$$

One interesting manifold among these exceptions is the sister of the figure- 8 complement $W(5)$. We will consider that manifold in detail in Section 12. 


\section{The figure-8 knot}

Here we prove:

10.1 Theorem Every non-trivial Dehn surgery on the figure-8 knot has virtual positive betti number.

Proof Let $M$ be the figure- 8 complement. As the figure- 8 knot is isotopic to its mirror image, the Dehn filling $M(p / q)$ is homeomorphic to $M(-p / q)$. Now, if $W$ is the Whitehead complement as in the last section, $M=W(-1)$. Hence $M$ has at least 6 interesting Seifert fibered surgeries namely $M( \pm 1), M( \pm 2)$ and $M( \pm 3)$. In (3), we saw exactly which orbifold quotients $\Gamma /\left\langle\mu^{n}\right\rangle$ arise when we try our method of transferring virtual positive betti number. By a minor miracle, Holt and Plesken have looked at exactly these quotients and shown:

10.2 Theorem [35] Let

$$
\begin{aligned}
& \Gamma_{n}^{1}=\left\langle a, b \mid a^{2}=b^{3}=(a b)^{7}=\left(a b a^{-1} b^{-1}\right)^{n}=1\right\rangle, \\
& \Gamma_{n}^{2}=\left\langle a, b \mid a^{2}=b^{4}=(a b)^{5}=\left(a b^{2}\right)^{n}\right\rangle, \text { and } \\
& \Gamma_{n}^{3}=\left\langle a, b \mid a^{3}=b^{3}=(a b)^{4}=\left(a b^{-1}\right)^{n}=1\right\rangle .
\end{aligned}
$$

These groups have virtual positive betti number if $n \geq 11$ for $\Gamma_{n}^{1}$ and $n \geq 6$ for $\Gamma_{n}^{2}$ and $\Gamma_{n}^{3}$.

Thus $M(\alpha)$ has virtual positive betti number if any of the following hold:

$$
\Delta(\alpha, \pm 1) \geq 11, \Delta(\alpha, \pm 2) \geq 6, \quad \text { or } \quad \Delta(\alpha, \pm 3) \geq 6 .
$$

It's easy to check that the only slopes $\alpha$ for which none of these hold are $\{\infty, 0, \pm 1, \pm 2\}$. Since $H_{1}(M(0))=\mathbb{Z}$ and the Seifert fibered manifolds $M( \pm 1)$ and $M( \pm 2)$ have virtual positive betti number, we've proved the theorem.

\section{Other groups of the form $\Gamma /\left\langle\gamma^{n}\right\rangle$ and further ques- tions}

As we have seen, groups of the form $\Gamma /\left\langle\gamma^{n}\right\rangle$, where $\Gamma$ is a Fuchsian group, are very useful for studying the Virtual Haken Conjecture via Dehn filling. So it is natural to ask: what other types of $\Gamma$ give similar results? In this section, we consider $\Gamma$ which are free products with amalgamation of finite groups. The 
key source here is Lubotzky's paper [40], which gives a number of applications of these groups to the Virtual Positive Betti Number Conjecture.

For convenience, we will only discuss free products with amalgamation, but there are analogous statements for HNN extensions. Let $\Gamma=A{ }{ }_{C} B$ be an amalgam of finite groups where $C$ is a proper subgroup of $A$ and $B$. The group $\Gamma$ acts on a tree $T$ with finite point stabilizers. By [50, $\S$ II.2.6], $\Gamma$ has a finite index subgroup $\Lambda$ which acts freely on $T$. The subgroup $\Lambda$ has to be free, and so $\Gamma$ is virtually free. It is not hard to show that if one of $[A: C]$ and $[B: C]$ is $\geq 3$ then $\Gamma$ is virtually a free group of rank $\geq 2$ [40, Lemma 2.2]. From now on, we will assume $[A: C] \geq 3$. Because $\Gamma$ is virtually free, it is natural to hope that the answer to the following question is yes:

11.1 Question Let $\Gamma$ be an amalgam of finite groups, and fix $\gamma \in \Gamma$ of infinite order. Does there exist an $N$ such that for all $n \geq N$, the group $\Gamma_{n}=\Gamma /\left\langle\gamma^{n}\right\rangle$ has virtual positive betti number?

Note that by Gromov, there is an $N$ such that $\Gamma_{n}$ is a non-elementary word hyperbolic group for all $n \geq N$.

Now consider these groups in the context of Dehn filling. Suppose $M$ is a manifold with torus boundary, and suppose $\alpha$ is a slope where $\pi_{1}(M(\alpha))$ surjects onto $\Gamma$, an amalgam of finite groups. Choose $\gamma$ in $\pi_{1}(\partial M)$ so that $\{\alpha, \gamma\}$ form a basis. The proof of Theorem 7.7 shows that if $M$ does not contain a closed incompressible surface, then the image of $\gamma$ in $\Gamma$ has infinite order.

There are candidate $\alpha$ where one expects that $\pi_{1}(M(\alpha))$ will surject onto an amalgam of finite groups. Suppose that $N=M(\alpha)$ contains a separating incompressible surface $S$. Then $\pi_{1}(N)$ splits as $\pi_{1}\left(N_{1}\right) *_{\pi_{1}(S)} \pi_{1}\left(N_{2}\right)$, where the $N_{i}$ are the components of $N \backslash S$. Recall that $\pi_{1}(S)$ is said to separable in $\pi_{1}(N)$ if it is closed in the profinite topology on $\pi_{1}(N)$. Lubotzky showed [40, Prop. 4.2] that if $\pi_{1}(S)$ is separable then there is a homomorphism from $\pi_{1}(N)$ to an amalgam of finite groups $\Gamma$, which respects the amalgam structure. Provided that $S$ is not a semi-fiber (that is, the $N_{i}$ are not both $I$-bundles), then $\Gamma=A *_{C} B$ can be chosen so that $[A: C] \geq 3$.

In general, we will say that $\pi_{1}(S)$ is weakly separable when there is such an amalgam preserving map from $\pi_{1}(N)$ to an amalgam of finite groups. A priori, this is weaker than $\pi_{1}(S)$ being closed in $\pi_{1}(N)$, which is in turn weaker than $\pi_{1}(N)$ being subgroup separable (aka LERF).

Note that if $\pi_{1}(S)$ is weakly separable, then $N$ has virtual positive betti number as $\pi_{1}(N)$ virtually maps onto a free group. If $N$ is hyperbolic, it seems quite 
possible that the fundamental group of an embedded surface is always weakly separable. If this is the case, there is no difference between being virtually Haken and having virtual positive betti number. Subgroup separability properties for 3 -manifold groups have been difficult to prove even in special cases. Weak separability also seems quite difficult to show even though the surface $S$ is embedded.

Let $M$ be a manifold with torus boundary which is hyperbolic. Assume that $M$ does not contain a closed incompressible surface. Then there are always at least two Dehn fillings of $M$ which contain an incompressible surface [14, 15]. If embedded surface subgroups are weakly separable, we would expect that for most $M$, there are at least two slopes where $\pi_{1}(M(\alpha))$ surjects onto an amalgam of finite groups. One has to say "most" here because $M(\alpha)$ might be a (semi-)fiber or the Poincaré conjecture might fail. This makes it plausible that, regardless of the truth of the virtual Haken conjecture in general, for a fixed $M$ all but finitely many Dehn fillings of $M$ have virtual positive betti number. In this context, it is worth mentioning the result of Cooper-Long [13] which says that for any such hyperbolic $M$ all but finitely many of the Dehn fillings contain a surface group. If fundamental groups of hyperbolic manifolds are subgroup separable, then this result would also imply that all but finitely many fillings of $M$ have virtual positive betti number.

One case where weak separability is known is when $N=M(\alpha)$ is irreducible and the incompressible surface $S$ in $N$ is a torus. Then $N$ is Haken and, by geometrization, $\pi_{1}(N)$ is residually finite. Using this it's not too hard to show that $\pi_{1}(S)$ is a separable subgroup. So in this case $\pi_{1}(N)$ maps to a amalgam of finite groups. In the next section, we will use these ideas in this special case to show that all of the Dehn filings on the sister of the figure- 8 complement satisfy the Virtual Haken Conjecture.

\section{The sister of the figure-8 complement}

Let $M$ be the sister of the figure- 8 complement. The manifold $M$ is the punctured torus bundle where the monodromy has trace -3 , and is also the surgery on the Whitehead link $W(5)$. We will use the basis $(\mu, \lambda)$ of $\pi_{1}(\partial M)$ coming from the standard basis on $W$. We will show:

12.1 Theorem Let $M$ be the sister of the figure- 8 complement. Then every Dehn filling of $M$ which has infinite fundamental group has virtual positive betti number. 
Proof The manifold $M$ has a self-homeomorphism which acts on $\pi_{1}(\partial M)$ via $(\mu, \lambda) \mapsto(\mu+\lambda,-\lambda)$. Let $N$ be the filling $M(4) \cong M(4 / 3)$. The manifold $N$ contains a separating incompressible torus. It turns out that this torus splits $N$ into a Seifert fibered space with base orbifold $D^{2}(2,3)$ and a twisted interval bundle over the Klein bottle. Rather than describe the details of this splitting, we will simply exhibit the final homomorphism from $\pi_{1}(N)$ onto an amalgam of finite groups. In fact, $\pi_{1}(N)$ surjects onto $\Gamma=S_{3}{ }^{*} C_{2} C_{4}$ where $C_{n}$ is a cyclic group of order $n$.

According to SnapPea, the group $\pi_{1}(N)$ has presentation:

$$
\left\langle a, b \mid a b^{2} a b^{-1} a^{3} b^{-1}=a b^{2} a^{-2} b^{2}=1\right\rangle
$$

where $\mu \in \pi(\partial M)$ becomes $a b$ in $\pi_{1}(N)$. If we add the relators $a^{3}=b^{4}=1$ to the presentation of $\pi_{1}(N)$, we get a surjection from $\pi_{1}(N)$ onto

$$
\Gamma=\left\langle a, b \mid a^{3}=b^{4}=\left(a b^{2}\right)^{2}=1\right\rangle .
$$

As $S_{3}$ has presentation $\left\langle x, y \mid x^{3}=y^{2}=(x y)^{2}=1\right\rangle$, we see that $\Gamma$ is $S_{3}{ }^{*} C_{2} C_{4}$ where the first factor is generated by $\left\{a, b^{2}\right\}$ and the second by $b$.

We will need:

12.2 Lemma Let $\Gamma$ be $S_{3} *_{C_{2}} C_{4}$ and let $\gamma \in \Gamma$ be $a b$. The group

$$
\Gamma_{n}=\Gamma /\left\langle\gamma^{n}\right\rangle
$$

has virtual positive betti number for all $n \geq 10$. For $n<10$, the group $\Gamma_{n}$ is finite.

Assuming the lemma, the theorem follows easily. Given a slope $\alpha$ in $\pi_{1}(\partial M)$, if either $\Delta(\alpha, 4) \geq 10$ or $\Delta(\alpha, 4 / 3) \geq 10$ then $M(\alpha)$ has virtual positive betti number. The only $\alpha$ which satisfy neither condition are $E=\{0,-1, \infty, 1$, $1 / 2,2,3,3 / 2,4,4 / 3,5 / 2,5 / 3,7 / 3,7 / 4\}$. One can check that the fillings along these slopes either have finite $\pi_{1}$ or have virtual positive betti number (the 6 hyperbolic fillings in $E$ are all among the census manifolds which we showed have virtual positive betti number in the earlier sections).

Now we will prove the lemma.

Proof of Lemma 12.2 As in the case of a Fuchsian group the key is to show:

12.3 Claim Let $n \geq 12$. Then there is a homomorphism $f$ from $\Gamma$ to a finite group $Q$ where $f$ is injective on the amalgam factors $S_{3}$ and $C_{4}$ and where $\gamma$ has order $n$. 
Assuming this claim, we will prove the theorem for $n \geq 12$. The Euler characteristic (in the sense of Wall [55]) of $\Gamma$ is $1 / 6+1 / 4-1 / 2=-1 / 12$. Let $K$ be the kernel of $f$. The subgroup $K$ is free, and from its Euler characteristic we see that it has rank $1+\# Q / 12$. Let $K^{\prime}$ be the kernel of the induced homomorphism from $\Gamma_{n} \rightarrow Q$. Then $H_{1}\left(K^{\prime}, \mathbb{Z}\right)$ is obtained from $H_{1}(K, \mathbb{Z})$ by adding $\# Q / n$ relators. As $n \geq 12$, this implies that $H_{1}\left(K^{\prime}, \mathbb{Z}\right)$ is infinite and $\Gamma_{n}$ has virtual positive betti number.

To prove the rest of the theorem, one can check that $\Gamma_{10}$ and $\Gamma_{11}$ have homomorphisms into $S_{12}$ and $\mathrm{PSL}_{2} \mathbb{F}_{23}$ respectively whose kernels have infinite $H_{1}$. Using coset enumeration, it is easy to check that $\Gamma_{n}$ is finite for $n<10$.

Now we establish the claim. For each $n$, we will inductively build a permutation representation $f: \Gamma \rightarrow S_{n}$ where $f(\gamma)$ has order $n$. We will say that $f: \Gamma \rightarrow S_{n}$ is special if it is faithful on the amalgam factors, $f(\gamma)$ is an $n$-cycle, and $f(b)$ fixes $n$. If $f$ satisfies these conditions except for $f(b)$ fixing $n$, we will say that $f$ is almost special. Our induction tool is:

12.4 Claim Suppose that $f$ is a special representation of $\Gamma$ into $S_{n}$. Then there exists a special representation of $\Gamma$ into $S_{n+6}$. Also, there exists an almost special representation of $\Gamma$ into $S_{n+7}$.

To see this, let $f$ be a special representation. First, we construct the representation into $S_{n+6}$. Let

$$
L=\{1,2, \ldots, n\} \cup\left\{p_{1}, p_{2}, p_{3}, p_{4}, p_{5}, p_{6}\right\} .
$$

We will find a special representation into $S_{L}$. Let $g: \Gamma \rightarrow S_{\left\{n, p_{1}, \ldots, p_{6}\right\}}$ be the special representation given by

$$
g(a)=\left(p_{1} p_{2} p_{3}\right)\left(p_{4} p_{5} p_{6}\right) \text { and } g(b)=\left(n p_{1}\right)\left(p_{2} p_{4} p_{3} p_{5}\right) .
$$

It's easy to check (using that $f(a)$ commutes with $g\left(b^{2}\right)$, etc.) that $h(a)=$ $f(a) g(a)$ and $h(b)=f(b) g(b)$ induces a homomorphism $h: \Gamma \rightarrow S_{L}$. Moreover, $h(a b)=f(a) g(a) f(b) g(b)=f(a) f(b) g(a) g(b)=f(a b) g(a b)$. Thus $h$ is the product of an $n$-cycle and a 7 -cycle which overlap only in $n$, and so is a $n+6$ cycle. So $h$ is special.

To construct the almost-special representation, do the same thing, where $g$ replaced is now defined by

$$
g(a)=\left(p_{1} p_{2} p_{3}\right)\left(p_{4} p_{5} p_{6}\right) \quad \text { and } \quad g(b)=\left(n p_{1}\right)\left(p_{2} p_{4} p_{3} p_{5}\right)\left(p_{6} p_{7}\right) .
$$

This establishes the inductive Claim 12.4. 
Using the induction, to prove Claim 12.3 it suffices to show that there are special representations for $n=6,7,15,17$, and that there is an almost-special representation for $n=16$. These are

$$
\begin{array}{rlrl}
n=6 & & a \mapsto(1,2,3)(4,5,6) \\
& b \mapsto(2,4,3,5) \\
n=7 & & a \mapsto(2,3,4)(5,6,7) \\
& b \mapsto(1,2)(3,5,4,6) \\
n=15 & & a \mapsto(2,3,4)(5,7,9)(6,8,11)(12,13,15) \\
& b \mapsto(1,2)(3,5,4,6)(7,10,11,14)(8,12,9,13) \\
n=16 & & a \mapsto(2,3,4)(5,7,9)(6,8,11)(12,13,15) \\
& b \mapsto(1,2)(3,5,4,6)(7,10,11,14)(8,12,9,13)(15,16) \\
n=17 & a \mapsto(2,3,5)(6,8,11)(7,10,9)(12,15,13)(14,16,17) \\
& b \mapsto(1,2,4,7)(3,6,9,12)(5,8,10,13)(11,14,15,16) .
\end{array}
$$

This completes the proof of the claim, the lemma, and thus the theorem.

\section{References}

[1] B Baumslag, S J Pride, Groups with two more generators than relators, J. London Math. Soc. 17 (1978) 425-426

[2] G Baumslag, J W Morgan, P B Shalen, Generalized triangle groups, Math. Proc. Cambridge Philos. Soc. 102 (1987) 25-31

[3] J S Birman, H M. Hilden, Heegaard splittings of branched coverings of $S^{3}$, Trans. Amer. Math. Soc. 213 (1975) 315-352

[4] W Bosma, J Cannon, Handbook of MAGMA functions, (1994)

[5] S Boyer, X Zhang, Virtual Haken 3-manifolds and Dehn filling, Topology 39 (2000) 103-114

[6] K S Brown, Buildings, Springer-Verlag, New York (1989)

[7] D Calegari, Promoting essential laminations, arXiv:math.GT/0210148

[8] P J Callahan, M V Hildebrand, J R Weeks, A census of cusped hyperbolic 3-manifolds, Math. Comp. 68 (1999) 321-332

[9] A Casson and D Jungreis, Convergence groups and Seifert fibered 3manifolds, Invent. Math. 118 (1994) 441-456

[10] S Choi and W Goldman, The deformation spaces of projectively flat structures on 2-orbifolds, preprint (2001) arXiv:math.GT/0107193

[11] J H Conway, R T Curtis, S P Norton, R A Parker, R A Wilson, Atlas of finite groups, Oxford University Press, Eynsham (1985) 
[12] D Cooper, D D Long, Virtually Haken Dehn-filling, J. Differential Geom. 52 (1999) 173-187

[13] D Cooper, D D Long, Some surface subgroups survive surgery, Geometry and Topology 5 (2001) 347-367

[14] M Culler, P B Shalen, Bounded, separating, incompressible surfaces in knot manifolds, Invent. Math. 75 (1984) 537-545

[15] M Culler, C M Gordon, J Luecke, P B Shalen, Dehn surgery on knots, Ann. of Math. 125 (1987) 237-300

[16] J D Dixon, Computing irreducible representations of groups, Math. Comp. 24 (1970) 707-712

[17] J D Dixon, Exact solution of linear equations using p-adic expansions, Numer. Math. 40 (1982) 137-141

[18] N M Dunfield, Alexander and Thurston norms of 3-manifolds fibering over the circle, Pacific J. Math 200 (2001) 43-58, arXiv:math.GT/9908050

[19] N M Dunfield, Which small volume hyperbolic 3-manifolds are Haken? Slides from a talk at the University of Warwick (1999) available from http://www . math. harvard.edu/ nathand

[20] N M Dunfield, W P Thurston, Data on the virtual Haken conjecture, http://www . computop.org/software/virtual_haken

[21] N M Dunfield, W P Thurston, Random 3-manifolds: Heuristics for the Virtual Haken Conjecture, in preparation

[22] B Fine, F Roehl, G Rosenberger, A Freiheitssatz for certain one-relator amalgamated products, In: "Combinatorial and geometric group theory (Edinburgh, 1993)", pages 73-86. Cambridge Univ. Press, 1995

[23] B Fine, G Rosenberger, Algebraic generalizations of discrete groups, Marcel Dekker Inc. New York (1999)

[24] B Freedman, MH Freedman, Kneser-Haken finiteness for bounded 3 manifolds locally free groups, and cyclic covers, Topology 37 (1998) 133-147

[25] D Gabai, Convergence groups are Fuchsian groups, Ann. of Math. 136 (1992) 447-510

[26] D Gabai, On the geometric and topological rigidity of hyperbolic 3-manifolds, J. Amer. Math. Soc. 10 (1997) 37-74

[27] D Gabai, R Meyerhoff, N Thurston, Homotopy hyperbolic 3-manifolds are hyperbolic, preprint, to appear in Ann. of Math.

[28] The GAP Group, Aachen, St Andrews, GAP - Groups, Algorithms, and Programming, Version 4.2, (2000) http://www-gap.dcs.st-and.ac.uk/〜gap

[29] O Goodman, Snap, http://www.ms.unimelb.edu.au/ snap

[30] A Hatcher, On the boundary curves of incompressible surfaces, Pacific J. Math. 99 (1982) 373-377 
[31] G Havas, D F Holt, S Rees, Recognizing badly presented $Z$-modules, Linear Algebra Appl. 192 (1993) 137-163

[32] G Havas, B S Majewski, Hermite normal form computation for integer matrices, from: "Proceedings of the Twenty-fifth Southeastern International Conference on Combinatorics, Graph Theory and Computing (Boca Raton, FL, 1994)", Congr. Numer. 105 (1994) 87-96

[33] G Havas, B S Majewski, Integer matrix diagonalization, J. Symbolic Comput. 24 (1997) 399-408

[34] M Hildebrand, J Weeks, A computer generated census of cusped hyperbolic 3-manifolds, from: "computers and mathematics (Cambridge, MA, 1989)", Springer (1989) 53-59

[35] D F Holt, W Plesken, A cohomological criterion for a finitely presented group to be infinite, J. London Math. Soc. (2) 45 (1992) 469-480

[36] J Hoste, M Thistlethwaite, Knotscape, www.math.utk.edu/ ${ }^{\text {morwen }}$

[37] W Jaco, U Oertel, An algorithm to decide if a 3 -manifold is a Haken manifold, Topology 23 (1984) 195-209

[38] R Kirby, Problems in low-dimensional topology, from: "Geometric topology (Athens, GA, 1993)", Amer. Math. Soc. Providence, RI (1997) 35-473, http://www. math . berkeley.edu/ “kirby/

[39] A Lubotzky, Eigenvalues of the Laplacian, the first Betti number and the congruence subgroup problem, Ann. of Math. 144 (1996) 441-452

[40] A Lubotzky, Free quotients and the first Betti number of some hyperbolic manifolds, Transform. Groups 1 (1996) 71-82

[41] W Meeks, L Simon, S-T Yau, Embedded minimal surfaces, exotic spheres, and manifolds with positive Ricci curvature, Ann. of Math. 116 (1982) 621-659

[42] G Mess, The Seifert fibered space conjecture and groups which are coarse quasiisometric to planes, preprint

[43] W Plesken, Presentations and representations of groups, from: "Algorithmic algebra and number theory (Heidelberg, 1997)", Springer, Berlin (1999) 423-434

[44] W Plesken, B Souvignier, Constructing rational representations of finite groups, Experiment. Math. 5 (1996) 39-47

[45] W Plesken, B Souvignier, Constructing representations of finite groups and applications to finitely presented groups, J. Algebra 202 (1998) 690-703

[46] L P Postnikova, A Schinzel, Primitive divisors of the expression $a^{n}-b^{n}$ in algebraic number fields, Mat. Sb. (N.S.) 75 (117) (1968) 171-177

[47] J D Rogawski, Automorphic representations of unitary groups in three variables, Princeton University Press, Princeton, NJ (1990)

[48] A Schinzel, Primitive divisors of the expression $A^{n}-B^{n}$ in algebraic number fields, J. Reine Angew. Math. 268/269 (1974) 27-33 
[49] P Scott, There are no fake Seifert fibre spaces with infinite $\pi_{1}$, Ann. of Math. (2) 117 (1983) 35-70

[50] J-P Serre, Trees, Springer-Verlag, Berlin (1980) translated from the French by John Stillwell

[51] C T Simpson, Products of matrices, from: "Differential geometry, global analysis, and topology (Halifax, NS, 1990)", Amer. Math. Soc. Providence, RI (1991) 157-185.

[52] C C Sims, Computation with finitely presented groups, Cambridge University Press, Cambridge (1994)

[53] W P. Thurston, The geometry and topology of 3-manifolds, Lecture notes (1978) http://www.msri.org/publications/books/gt3m/

[54] F Waldhausen, The word problem in fundamental groups of sufficiently large irreducible 3-manifolds, Ann. of Math. 88 (1968) 272-280

[55] C T C Wall, Rational Euler characteristics, Proc. Cambridge Philos. Soc. 57 (1961) 182-184

[56] J Weeks, SnapPea, http://www.geometrygames.org/

[57] Y Q Wu, Incompressibility of surfaces in surgered 3-manifolds, Topology 31 (1992) 271-279 


\section{Appendix}

\begin{tabular}{|c|c|c|c|c|c|c|c|c|c|c|c|c|}
\hline & $A_{5}$ & $L_{2}(7)$ & $A_{6}$ & $L_{2}(8)$ & $L_{2}(11)$ & $L_{2}(13)$ & $L_{2}(17)$ & $A_{7}$ & $L_{2}(19)$ & $L_{2}(16)$ & $L_{3}(3)$ & $U_{3}(3)$ \\
\hline$A_{5}$ & 1.00 & 0.02 & 0.13 & 0.05 & 0.17 & 0.03 & -0.03 & 0.12 & 0.15 & 0.09 & 0.02 & 0.02 \\
\hline$L_{2}(7)$ & 0.02 & 1.00 & 0.04 & 0.23 & 0.05 & 0.16 & 0.05 & 0.06 & -0.02 & -0.04 & 0.12 & 0.09 \\
\hline$A_{6}$ & 0.13 & 0.04 & 1.00 & -0.04 & 0.13 & -0.07 & 0.02 & 0.10 & 0.11 & 0.09 & 0.04 & 0.00 \\
\hline$L_{2}(8)$ & 0.05 & 0.23 & -0.04 & 1.00 & 0.02 & 0.20 & 0.06 & 0.08 & 0.05 & -0.00 & -0.00 & 0.11 \\
\hline$L_{2}(11)$ & 0.17 & 0.05 & 0.13 & 0.02 & 1.00 & -0.01 & 0.03 & 0.11 & 0.11 & 0.14 & 0.07 & 0.05 \\
\hline$L_{2}(13)$ & 0.03 & 0.16 & -0.07 & 0.20 & -0.01 & 1.00 & 0.00 & -0.01 & 0.04 & 0.04 & 0.06 & 0.09 \\
\hline$L_{2}(17)$ & -0.03 & 0.05 & 0.02 & 0.06 & 0.03 & 0.00 & 1.00 & 0.01 & 0.05 & 0.03 & 0.11 & 0.12 \\
\hline & 0.12 & 0.06 & 0.10 & 0.08 & 0.11 & -0.01 & 0.01 & 1.00 & 0.08 & 0.10 & 0.03 & 0.11 \\
\hline$L_{2}(19)$ & 0.15 & -0.02 & 0.11 & 0.05 & 0.11 & 0.04 & 0.05 & 0.08 & 1.00 & 0.11 & 0.03 & 0.03 \\
\hline$L_{2}(16)$ & 0.09 & -0.04 & 0.09 & -0.00 & 0.14 & 0.04 & 0.03 & 0.10 & 0.11 & 1.00 & -0.02 & 0.07 \\
\hline$L_{3}(3)$ & 0.02 & 0.12 & 0.04 & -0.00 & 0.07 & 0.06 & 0.11 & 0.03 & 0.03 & -0.02 & 1.00 & 0.10 \\
\hline$U_{3}(3)$ & 0.02 & 0.09 & 0.00 & 0.11 & 0.05 & 0.09 & 0.12 & 0.11 & 0.03 & 0.07 & 0.10 & 1.00 \\
\hline$L_{2}(23)$ & 0.01 & 0.10 & 0.03 & 0.07 & 0.05 & 0.03 & 0.12 & -0.04 & 0.03 & 0.03 & 0.15 & 0.04 \\
\hline$L_{2}(25)$ & 0.04 & 0.06 & 0.15 & 0.06 & 0.14 & 0.03 & 0.13 & 0.09 & 0.10 & 0.10 & 0.21 & 0.08 \\
\hline$M_{11}$ & 0.16 & 0.03 & 0.21 & -0.00 & 0.09 & -0.02 & 0.09 & 0.12 & 0.01 & 0.05 & 0.05 & 0.06 \\
\hline$L_{2}(27)$ & -0.01 & 0.19 & -0.05 & 0.29 & 0.02 & 0.15 & 0.04 & 0.09 & 0.04 & 0.00 & 0.06 & $\overline{0.10}$ \\
\hline$L_{2}(29)$ & 0.01 & 0.13 & 0.01 & 0.14 & 0.17 & 0.10 & -0.00 & 0.19 & 0.15 & 0.06 & 0.00 & 0.01 \\
\hline$L_{2}(31)$ & 0.08 & 0.08 & 0.18 & 0.00 & 0.10 & -0.05 & 0.11 & 0.04 & 0.10 & 0.09 & 0.09 & 0.06 \\
\hline & 0.11 & 0.14 & 0.12 & 0.11 & 0.08 & 0.08 & 0.07 & 0.17 & 0.10 & 0.07 & 0.04 & 0.11 \\
\hline$L_{3}(4)$ & 0.15 & 0.03 & 0.13 & 0.02 & 0.11 & -0.04 & 0.03 & 0.23 & 0.05 & 0.01 & 0.07 & 0.03 \\
\hline$L_{2}(37)$ & 0.02 & 0.01 & 0.06 & 0.02 & 0.06 & 0.02 & 0.07 & 0.04 & 0.08 & 0.13 & 0.00 & 0.02 \\
\hline$U_{4}(2)$ & 0.18 & 0.02 & 0.24 & -0.00 & 0.07 & -0.04 & -0.01 & 0.13 & 0.05 & 0.05 & 0.02 & -0.01 \\
\hline $\mathrm{Sz}(8)$ & -0.00 & 0.02 & 0.11 & -0.01 & 0.03 & -0.03 & 0.00 & -0.02 & 0.09 & -0.03 & -0.01 & -0.03 \\
\hline$L_{2}(32)$ & 0.07 & 0.06 & -0.02 & -0.02 & 0.01 & 0.03 & 0.00 & -0.02 & 0.01 & 0.02 & -0.00 & 0.05 \\
\hline
\end{tabular}

\begin{tabular}{|c|c|c|c|c|c|c|c|c|c|c|c|c|}
\hline & $L_{2}(23)$ & $L_{2}(25)$ & $M_{11}$ & $L_{2}(27)$ & $L_{2}(29)$ & $L_{2}(31)$ & $A_{8}$ & $L_{3}(4)$ & $L_{2}(37)$ & $U_{4}(2)$ & $\mathrm{Sz}(8)$ & $L_{2}(32)$ \\
\hline$A_{5}$ & 0.01 & 0.04 & 0.16 & -0.01 & 0.01 & 0.08 & 0.11 & 0.15 & 0.02 & 0.18 & -0.00 & 0.07 \\
\hline$L_{2}(7)$ & 0.10 & 0.06 & 0.03 & 0.19 & 0.13 & 0.08 & 0.14 & 0.03 & 0.01 & 0.02 & 0.02 & 0.06 \\
\hline$A_{6}$ & 0.03 & 0.15 & 0.21 & -0.05 & 0.01 & 0.18 & 0.12 & 0.13 & 0.06 & 0.24 & 0.11 & -0.02 \\
\hline$L_{2}(8)$ & 0.07 & 0.06 & -0.00 & 0.29 & 0.14 & 0.00 & 0.11 & 0.02 & 0.02 & -0.00 & -0.01 & -0.02 \\
\hline$L_{2}(11)$ & 0.05 & 0.14 & 0.09 & 0.02 & 0.17 & 0.10 & 0.08 & 0.11 & 0.06 & 0.07 & 0.03 & 0.01 \\
\hline$L_{2}(13)$ & 0.03 & 0.03 & -0.02 & 0.15 & 0.10 & -0.05 & 0.08 & -0.04 & 0.02 & -0.04 & -0.03 & 0.03 \\
\hline$\overline{L_{2}(17)}$ & 0.12 & 0.13 & 0.09 & 0.04 & -0.00 & 0.11 & 0.07 & 0.03 & 0.07 & -0.01 & 0.00 & 0.00 \\
\hline & -0.04 & 0.09 & 0.12 & 0.09 & 0.19 & 0.04 & 0.17 & 0.23 & 0.04 & 0.13 & -0.02 & -0.02 \\
\hline$L_{2}(19)$ & 0.03 & 0.10 & 0.01 & 0.04 & 0.15 & 0.10 & 0.10 & 0.05 & 0.08 & 0.05 & 0.09 & 0.01 \\
\hline$\overline{L_{2}(16)}$ & 0.03 & $\overline{0.10}$ & 0.05 & 0.00 & 0.06 & $\overline{0.09}$ & 0.07 & 0.01 & 0.13 & $\overline{0.05}$ & -0.03 & 0.02 \\
\hline$L_{3}(3)$ & 15 & 21 & 0.05 & 0.06 & 0.00 & 0.09 & 0.04 & 0.07 & 0.00 & 0.02 & -0.01 & -0.00 \\
\hline$U_{3}(3)$ & 0.04 & 0.08 & 0.06 & 0.10 & 0.01 & 0.06 & 0.11 & 0.03 & 0.02 & -0.01 & -0.03 & 0.05 \\
\hline$L_{2}(23)$ & 1.00 & 0.09 & 0.04 & 0.07 & 0.02 & 0.08 & -0.02 & 0.01 & 0.00 & 0.01 & -0.04 & 0.08 \\
\hline$L_{2}(25)$ & 09 & 1.00 & 0.05 & 0.15 & 0.07 & 0.14 & 0.12 & 0.05 & 0.06 & 0.10 & 0.03 & 0.03 \\
\hline$M_{11}$ & 0.04 & 0.05 & 1.00 & -0.01 & -0.00 & 0.14 & 0.14 & 0.19 & 0.00 & 0.21 & 0.09 & 0.04 \\
\hline$\overline{L_{2}(27)}$ & 0.07 & 0.15 & -0.01 & 1.00 & 0.19 & $\overline{0.01}$ & $\overline{0.11}$ & $\overline{0.02}$ & 0.03 & -0.01 & -0.04 & 0.05 \\
\hline$L_{2}(29)$ & & & -0.00 & 0.19 & 1.00 & 0.07 & 0.12 & 0.1 & 0.08 & 0.03 & -0.01 & -0.02 \\
\hline$L_{2}(31)$ & 0.08 & 0.14 & 0.14 & 0.01 & 0.07 & 1.00 & 0.09 & 0.10 & 0.02 & 0.13 & 0.08 & 0.08 \\
\hline$A_{8}$ & -0.02 & 0.12 & 0.14 & 0.11 & 0.12 & 0.09 & 1.00 & 0.15 & -0.01 & 0.14 & 0.08 & -0.03 \\
\hline$L_{3}(4)$ & 0.01 & & 0.19 & 0.02 & & & 0.15 & & $-0 .($ & 0.21 & 0.26 & -0.04 \\
\hline$L_{2}(37)$ & 0.00 & 0.06 & 0.00 & 0.03 & 0.08 & 0.02 & -0.01 & -0.00 & 1.00 & 0.01 & -0.03 & 0.06 \\
\hline$U_{4}(2)$ & 0.01 & 0.10 & 0.21 & -0.01 & 0.03 & 0.13 & 0.14 & 0.21 & 0.01 & 1.00 & 0.02 & 0.04 \\
\hline $\mathrm{Sz}(8)$ & -0.04 & .03 & 0.09 & -0.04 & -0.01 & 0.0 & 0.08 & 0.26 & -0.03 & 0.02 & 1.00 & -0.03 \\
\hline$L_{2}(32)$ & 0.08 & 0.03 & 0.04 & 0.05 & -0.02 & 0.08 & -0.03 & -0.04 & 0.06 & 0.04 & -0.03 & 1.00 \\
\hline
\end{tabular}

Table 5: This table gives the correlations between: (having a cover with group 1, having a cover with group 2). The average off-diagonal correlation is 0.06 . 


\begin{tabular}{|c|c|c|c|c|c|c|c|c|c|c|c|c|}
\hline & $A_{5}$ & $L_{2}(7)$ & $A_{6}$ & $L_{2}(8)$ & $L_{2}(11)$ & $L_{2}(13)$ & $L_{2}(17)$ & $A_{7}$ & $L_{2}(19)$ & $L_{2}(16)$ & $L_{3}(3)$ & $U_{3}(3)$ \\
\hline$A_{5}$ & 1.00 & -0.01 & 0.28 & 0.05 & 0.25 & 0.01 & 0.06 & 0.11 & 0.23 & 0.11 & 0.02 & 0.02 \\
\hline$L_{2}(7)$ & -0.01 & 1.00 & 0.05 & 0.38 & 0.04 & 0.25 & 0.14 & 0.11 & 0.02 & 0.01 & 0.17 & 0.13 \\
\hline$A_{6}$ & 0.28 & 0.05 & 1.00 & 0.00 & 0.22 & -0.07 & 0.12 & 0.13 & 0.17 & 0.10 & 0.08 & 0.02 \\
\hline$L_{2}(8)$ & 0.05 & 0.38 & 0.00 & 1.00 & 0.05 & 0.36 & 0.11 & 0.12 & 0.06 & 0.06 & 0.03 & 0.12 \\
\hline$L_{2}(11)$ & 0.25 & 0.04 & 0.22 & 0.05 & 1.00 & 0.03 & 0.07 & 0.06 & 0.18 & 0.12 & 0.08 & 0.04 \\
\hline$L_{2}(13)$ & 0.01 & 0.25 & -0.07 & 0.36 & 0.03 & 1.00 & 0.07 & 0.01 & 0.04 & 0.10 & 0.08 & 0.13 \\
\hline$L_{2}(17)$ & 0.06 & 0.14 & 0.12 & 0.11 & 0.07 & 0.07 & 1.00 & 0.07 & 0.12 & 0.07 & 0.15 & 0.11 \\
\hline$A_{7}$ & 0.11 & 0.11 & 0.13 & 0.12 & 0.06 & 0.01 & 0.07 & 1.00 & 0.07 & 0.09 & 0.07 & 0.13 \\
\hline$L_{2}(19)$ & 0.23 & 0.02 & 0.17 & 0.06 & 0.18 & 0.04 & 0.12 & 0.07 & 1.00 & 0.09 & 0.08 & 0.05 \\
\hline$L_{2}(16)$ & 0.11 & 0.01 & 0.10 & 0.06 & 0.12 & 0.10 & 0.07 & 0.09 & 0.09 & 1.00 & 0.03 & 0.10 \\
\hline$L_{3}(3)$ & 0.02 & 0.17 & 0.08 & 0.03 & 0.08 & 0.08 & 0.15 & 0.07 & 0.08 & 0.03 & 1.00 & 0.14 \\
\hline$U_{3}(3)$ & 0.02 & 0.13 & 0.02 & 0.12 & 0.04 & 0.13 & 0.11 & 0.13 & 0.05 & 0.10 & 0.14 & 1.00 \\
\hline$\overline{L_{2}(23)}$ & 0.06 & 0.13 & 0.02 & 0.04 & 0.06 & 0.05 & 0.13 & -0.01 & 0.06 & 0.05 & 0.15 & 0.09 \\
\hline$L_{2}(25)$ & 0.12 & 0.13 & 0.20 & 0.14 & 0.17 & 0.06 & 0.17 & 0.12 & 0.14 & 0.15 & 0.21 & 0.13 \\
\hline$M_{11}$ & 0.19 & 0.04 & 0.33 & 0.03 & 0.12 & 0.00 & 0.11 & 0.17 & 0.07 & 0.08 & 0.06 & 0.07 \\
\hline$L_{2}(27)$ & -0.03 & 0.38 & -0.06 & 0.45 & 0.05 & 0.35 & 0.06 & 0.10 & 0.01 & 0.01 & 0.09 & 0.16 \\
\hline$L_{2}(29)$ & 0.08 & 0.17 & 0.04 & 0.24 & 0.24 & 0.18 & 0.02 & 0.22 & 0.15 & 0.05 & 0.06 & 0.03 \\
\hline$L_{2}(31)$ & 0.22 & 0.08 & 0.30 & 0.02 & 0.15 & 0.02 & 0.24 & 0.08 & 0.15 & 0.09 & 0.15 & 0.08 \\
\hline & 0.11 & 0.15 & 0.15 & 0.14 & 0.08 & 0.12 & 0.14 & 0.20 & 0.08 & 0.09 & 0.09 & 0.12 \\
\hline$L_{3}(4)$ & 0.21 & 0.08 & 0.27 & 0.04 & 0.15 & -0.01 & 0.05 & 0.28 & 0.13 & 0.09 & 0.11 & 0.04 \\
\hline$L_{2}(37)$ & 0.09 & 0.03 & 0.12 & 0.05 & 0.14 & 0.10 & 0.15 & 0.02 & 0.09 & 0.16 & 0.03 & 0.08 \\
\hline $\bar{U}_{4}(2)$ & 0.17 & 0.03 & 0.34 & 0.01 & 0.10 & -0.01 & 0.05 & 0.15 & 0.10 & 0.08 & 0.05 & 0.02 \\
\hline $\mathrm{Sz}(8)$ & 0.08 & 0.08 & 0.17 & 0.03 & 0.06 & 0.01 & 0.05 & 0.04 & 0.10 & 0.03 & 0.02 & -0.01 \\
\hline$L_{2}(32)$ & 0.06 & 0.05 & -0.01 & 0.01 & 0.05 & 0.06 & 0.02 & -0.01 & 0.04 & 0.05 & 0.01 & 0.08 \\
\hline
\end{tabular}

\begin{tabular}{|c|c|c|c|c|c|c|c|c|c|c|c|c|}
\hline & $L_{2}(23)$ & $L_{2}(25)$ & $M_{11}$ & $L_{2}(27)$ & $L_{2}(29)$ & $L_{2}(31)$ & $A_{8}$ & $L_{3}(4)$ & $L_{2}(37)$ & $U_{4}(2)$ & $\mathrm{Sz}(8)$ & $L_{2}(32)$ \\
\hline & 0.06 & 0.12 & 0.19 & -0.03 & 0.08 & 0.22 & 0.11 & 0.21 & 0.09 & 0.17 & 0.08 & 0.06 \\
\hline$L_{2}(7)$ & 0.13 & 0.13 & 0.04 & 0.38 & 0.17 & 0.08 & 0.15 & 0.08 & 0.03 & 0.03 & 0.08 & 0.05 \\
\hline$A_{6}$ & 0.02 & 0.20 & 0.33 & -0.06 & 0.04 & 0.30 & 0.15 & 0.27 & 0.12 & 0.34 & 0.17 & -0.01 \\
\hline$L_{2}(8)$ & 0.04 & 0.14 & 0.03 & 0.45 & 0.24 & 0.02 & 0.14 & 0.04 & 0.05 & 0.01 & 0.03 & 0.01 \\
\hline$L_{2}(11)$ & 0.06 & 0.17 & 0.12 & 0.05 & 0.24 & 0.15 & 0.08 & 0.15 & 0.14 & 0.10 & 0.06 & 0.05 \\
\hline$L_{2}(13)$ & 0.05 & 0.06 & 0.00 & 0.35 & 0.18 & 0.02 & 0.12 & -0.01 & 0.10 & -0.01 & 0.01 & 0.06 \\
\hline$L_{2}(17)$ & 0.13 & 0.17 & 0.11 & 0.06 & 0.02 & 0.24 & 0.14 & 0.05 & 0.15 & 0.05 & 0.05 & 0.02 \\
\hline & -0.01 & 0.12 & 0.17 & 0.10 & 0.22 & 0.08 & 0.20 & 0.28 & 0.02 & 0.15 & 0.04 & -0.01 \\
\hline$L_{2}(19)$ & 0.06 & 0.14 & 0.07 & 0.01 & 0.15 & 0.15 & 0.08 & 0.13 & 0.09 & 0.10 & 0.10 & 0.04 \\
\hline$L_{2}(16)$ & 0.05 & 0.15 & 0.08 & 0.01 & 0.05 & 0.09 & 0.09 & 0.09 & 0.16 & 0.08 & 0.03 & 0.05 \\
\hline$L_{3}(3)$ & 0.15 & 0.21 & 0.06 & 0.09 & 0.06 & 0.15 & 0.09 & 0.11 & 0.03 & 0.05 & 0.02 & 0.01 \\
\hline$U_{3}(3)$ & 0.09 & 0.13 & 0.07 & 0.16 & 0.03 & 0.08 & 0.12 & 0.04 & 0.08 & 0.02 & -0.01 & 0.08 \\
\hline$\overline{L_{2}(23)}$ & 1.00 & 0.11 & 0.05 & 0.04 & 0.04 & 0.08 & 0.01 & 0.04 & 0.09 & 0.02 & -0.05 & 0.15 \\
\hline$L_{2}(25)$ & 0.11 & 1.00 & 0.12 & 0.15 & 0.15 & 0.18 & 0.20 & 0.06 & 0.16 & 0.14 & 0.04 & 0.02 \\
\hline$M_{11}$ & 0.05 & 0.12 & 1.00 & -0.04 & 0.02 & 0.22 & 0.14 & 0.24 & 0.05 & 0.25 & 0.08 & 0.05 \\
\hline$L_{2}(27)$ & 0.04 & & -0.04 & 1.00 & 0.25 & -0.03 & 0.10 & 0.02 & 0.03 & 0.01 & 0.01 & 0.08 \\
\hline$L_{2}(29)$ & 0.04 & 0.15 & 0.02 & 0.25 & 1.00 & 0.06 & 0.13 & 0.21 & 0.12 & 0.04 & 0.04 & 0.01 \\
\hline$L_{2}(31)$ & 0.08 & 0.18 & 0.22 & -0.03 & 0.06 & 1.00 & 0.10 & 0.12 & 0.12 & 0.15 & 0.10 & 0.06 \\
\hline & 0.01 & 0.20 & 0.14 & 0.10 & 0.13 & 0.10 & 1.00 & 0.18 & 0.07 & 0.16 & 0.09 & -0.03 \\
\hline$L_{3}(4)$ & 0.04 & 0.06 & 0.24 & 0.02 & 0.21 & 0.12 & 0.18 & 1.00 & 0.02 & 0.25 & 0.30 & -0.04 \\
\hline$L_{2}(37)$ & 0.09 & 0.16 & 0.05 & 0.03 & 0.12 & 0.12 & 0.07 & 0.02 & 1.00 & 0.06 & 0.03 & 0.10 \\
\hline$U_{4}(2)$ & 0.02 & & 0.25 & 0.01 & 0.04 & & 0.16 & 0.25 & 0.06 & 1.00 & -0.01 & 0.01 \\
\hline $\mathrm{Sz}(8)$ & -0.05 & 0.04 & 0.08 & 0.01 & 0.04 & 0.10 & 0.09 & 0.30 & 0.03 & -0.01 & 1.00 & -0.07 \\
\hline$L_{2}(32)$ & 0.15 & 0.02 & 0.05 & 0.08 & 0.01 & 0.06 & -0.03 & -0.04 & 0.10 & 0.01 & -0.07 & 1.00 \\
\hline
\end{tabular}

Table 6: This table gives the correlations between: (having a cover with group 1 with positive betti number, having a cover with group 2 with positive betti number). The average off-diagonal correlation is 0.09 . 